\title{
FAILURE OF CANCELLATION FOR DIRECT SUMS OF LINE BUNDLES
}

\author{
RICHARD G. SWAN
}

\begin{abstract}
In answer to a question of Murthy and Wiegand, examples are given of finitely generated projective modules $L$ of rank 1 over a commutative ring $R$ such that $L \oplus L^{-1}$ is stably free but not free.

Examples are also given of projective modules for which the determinant map det: $\operatorname{Aut}(P) \rightarrow R^{*}$ is not onto. Some related topological questions are also discussed.
\end{abstract}

The following question was raised independently by M. P. Murthy and R. Wiegand.

Question. Does there exist a commutative ring $R$ and a finitely generated projective module $L$ over $R$ of rank 1 such that $L \oplus L^{-1}$ is stably free but not free?

I will show here that the answer is affirmative. We can even assume that $L \oplus L^{-1} \oplus R$ is free.

Theorem 1. There exist rings $R$ of the following types with a finitely generated projective module $L$ over $R$ of rank 1 such that $L \oplus L^{-1} \oplus R$ is free but $L \oplus L^{-1}$ is not free.

(1) $R$ is a sub $\mathbb{R}$-algebra of $\mathbb{R}[x, y]$, finitely generated over $\mathbb{R}$, and with quotient field $\mathbb{R}(x, y)$.

(2) $R$ is a smooth rational domain of dimension 3, finitely generated over $\mathbb{R}$.

(3) $R$ is a rational domain of dimension 5, finitely generated over $\mathbb{C}$.

(4) $R$ is a smooth rational domain of dimension 7, finitely generated over $\mathbb{C}$.

The examples will be constructed explicitly but the proof that $L \oplus L^{-1}$ is not free will use topological methods as in [SV]. In $\S \S 7$ and 8 , we give examples of projective modules $P$ such that the determinant map det: $\operatorname{Aut}(P) \rightarrow R^{*}$ is not onto.

All topological spaces considered here will be assumed to have the homotopy type of $C W$-complexes.

Received by the editors November 29, 1990.

1980 Mathematics Subject Classification (1985 Revision). Primary 13C10; Secondary 14F05, $55 \mathrm{R} 25$. 


\section{1. $S O(n)$ AND $S U(n)$ BUNDLES}

It is well known that real and complex vector bundles can be thought of as $O(n)$ and $U(n)$-bundles since the inclusions $O(n) \subset G L_{n}(\mathbb{R})$ and $U(n) \subset$ $G L_{n}(\mathbb{C})$ are homotopy equivalences. In our construction, it will be more convenient to consider $S O(n)$ and $S U(n)$-bundles. These are equivalent to $S L_{n}(\mathbb{R})$ and $S L_{n}(\mathbb{C})$-bundles, i.e., to vector bundles with a continuously varying choice of volume element on each fiber or, equivalently, to vector bundles $E$ with a chosen trivialization of $\bigwedge^{n} E$ where $n=\operatorname{rk} E$. Such bundles over a space $X$ are classified by the sets of homotopy classes $[X, B S O(n)]$ and $[X, B S U(n)]$ while vector bundles are classified by $[X, B O(n)]$ and $[X, B U(n)]$. See [HFB, SFB]. Here, free homotopies are considered rather than homotopies preserving a basepoint (i.e., we are working in the category of spaces without basepoint). This distinction is unnecessary for $B S O(n), B S U(n)$, and $B U(n)$ since these spaces are simply connected (see Corollary 9.2).

The maps $[X, B S O(n)] \rightarrow[X, B O(n)]$ and $[X, B S U(n)] \rightarrow[X, B U(n)]$ correspond to forgetting the choice of volume element. It is well known that the image of $[X, B S O(n)] \rightarrow[X, B O(n)]$ consists of those vector bundles $E$ with $w_{1}(E)=0$ while the image of $[X, B S U(n)] \rightarrow[X, B U(n)]$ consists of those vector bundles $E$ with $c_{1}(E)=0$. This follows easily from the fibrations $B S O(n) \rightarrow B O(n) \rightarrow B\{ \pm 1\}$ and $B S U(n) \rightarrow B U(n) \rightarrow B S^{1}$.

Let $*$ denote the trivial homotopy class.

Lemma 1.1. The inverse image of * under $[X, B S O(n)] \rightarrow[X, B O(n)]$ or $[X, B S U(n)] \rightarrow[X, B U(n)]$ consists of * alone.

Proof. The fibrations $\{ \pm 1\} \rightarrow B O(n) \rightarrow B S O(n)$ and $S^{1} \rightarrow B U(n) \rightarrow B S U(n)$ show that the inverse images in question are the images of the maps $[X,\{ \pm 1\}]$ $\rightarrow[X, B O(n)]$ and $\left[X, S^{1}\right] \rightarrow[X, B U(n)]$. These are trivial since the maps $\{ \pm 1\} \rightarrow B O(n)$ and $S^{1} \rightarrow B U(n)$ are homotopic to constant maps.

It follows that the triviality or nontriviality of the corresponding bundles can be decided by looking at $[X, B S O(n)]$ and $[X, B S U(n)]$ themselves.

Remark. In spite of this result, the maps $[X, B S O(n)] \rightarrow[X, B O(n)]$ and $[X, B S U(n)] \rightarrow[X, B U(n)]$ are not injective in general. Examples will be given in $\S \S 7$ and 8 .

\section{REAL BUNDLES}

Real line bundles $L$ are classified by maps into $B C_{2}$ where $C_{2}=\{ \pm 1\}$ is the cyclic group of order 2 . For real line bundles , $L^{-1} \approx L$, and the functor taking $L$ to $L \oplus L^{-1}$ is given by the map $\left[X, B C_{2}\right] \rightarrow[X, B S O(2)]$ which is induced by the homomorphism $C_{2} \rightarrow S O(2)$ sending -1 to

$$
\left(\begin{array}{cc}
-1 & 0 \\
0 & -1
\end{array}\right)
$$

Let $\mathscr{O}$ denote the trivial line bundle. Then the functor taking $L$ to $L \oplus$ $L^{-1} \oplus \mathscr{O}$ is given by the map $\left[X, B C_{2}\right] \rightarrow[X, B S O(3)]$ which is induced by the homomorphism $C_{2} \rightarrow S O(3)$ sending -1 to the matrix 


$$
J=\left(\begin{array}{ccc}
1 & 0 & 0 \\
0 & -1 & 0 \\
0 & 0 & -1
\end{array}\right)
$$

Therefore, the universal example for line bundles $L$ with $\mathscr{O} \oplus L \oplus L^{-1}$ trivial is the fiber $X=S O(3) / C_{2}$ of the map $B C_{2} \rightarrow B S O(3)$. Let $L$ be the line bundle on $X$ induced by the map $X \rightarrow B C_{2}$ or, equivalently, the line bundle associated with the principal fibration $S O(3) \rightarrow X$. It remains to show that $L \oplus L^{-1}$ is nontrivial.

Recall that a cohomology class $\alpha \in H^{n}(Y, G)$ is called spherical if there is a map $Y \rightarrow S^{n}$ such that $\alpha$ lies in the image of $H^{n}\left(S^{n}, G\right) \rightarrow H^{n}(Y, G)$. Since $H^{1}(Y, \mathbb{Z})=\left[Y, S^{1}\right]$, a class $\alpha \in H^{1}(Y, \mathbb{Z} / 2 \mathbb{Z})$ is spherical if and only if $\alpha$ lies in the image of $H^{1}(Y, \mathbb{Z}) \rightarrow H^{1}(Y, \mathbb{Z} / 2 \mathbb{Z})$.

Lemma 2.1. Let $L$ be a real line bundle on $Y$ given by a class $\alpha \in H^{1}(Y, \mathbb{Z} / 2 \mathbb{Z})$ $=\left[Y, B C_{2}\right]$. Then $L \oplus L^{-1}$ is trivial if and only if $\alpha$ is spherical.

Proof. If $L \oplus L^{-1}$ is trivial, the composition $Y \rightarrow B C_{2} \rightarrow B S O(2)$ is homotopic to a constant map so $Y \rightarrow B C_{2}$ factors through the fiber $S O(2) / C_{2}=S^{1}$ of the map $B C_{2} \rightarrow B S O(2)$. For the converse, we first observe that the homotopy sequence of the fibration $S^{1} \rightarrow B C_{2} \rightarrow B S O(2)$ shows that $\mathbb{Z}=\pi_{1}\left(S^{1}\right) \rightarrow$ $\pi_{1}\left(B C_{2}\right)=\mathbb{Z} / 2 \mathbb{Z}$ is onto. Therefore $H^{1}\left(B C_{2}, \mathbb{Z} / 2 \mathbb{Z}\right) \rightarrow H^{1}\left(S^{1}, \mathbb{Z} / 2 \mathbb{Z}\right)$ is an isomorphism. If $\alpha$ is spherical, let $f: Y \rightarrow S^{1}$ such that $\alpha$ is the image of the generator of $H^{1}\left(S^{1}, \mathbb{Z} / 2 \mathbb{Z}\right)$ under the induced map $f^{*}: H^{1}\left(S^{1}, \mathbb{Z} / 2 \mathbb{Z}\right) \rightarrow$ $H^{1}(Y, \mathbb{Z} / 2 \mathbb{Z})$. Since $H^{1}(Y, \mathbb{Z} / 2 \mathbb{Z})=\left[Y, B C_{2}\right]$, the diagram

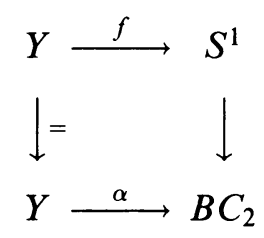

commutes up to homotopy showing that $\alpha$ is homotopic to a map into the fiber and so the composition $Y \rightarrow B C_{2} \rightarrow B S O(2)$ is homotopic to a constant map.

Corollary 2.2. Let $L$ be a real line bundle on $Y$. If $H^{1}(Y, \mathbb{Z})=0$ and $L \oplus L^{-1}$ is trivial, then $L$ is trivial.

Now the universal covering of $S O(3)$ is $S^{3} \rightarrow S O(3)$ sending a unit quaternion $q$ to the map $x \rightarrow q x q^{-1}$ on $\mathbb{R} i+\mathbb{R} j+\mathbb{R} k$. This map sends $i$ to the matrix $J$ above so we see that the universal covering of $X$ is $S^{3} \rightarrow S^{3} /\langle i\rangle=X$ In particular, $\pi_{1}(X)=\mathbb{Z} / 4 \mathbb{Z}$ showing that $H^{1}(X, \mathbb{Z})=\operatorname{Hom}\left(\pi_{1}(X), \mathbb{Z}\right)=0$. The homotopy sequence $\cdots \rightarrow \pi_{1} X \rightarrow \pi_{1} B C_{2} \rightarrow \pi_{1} B S O(3)=0$ of the fibration $X \rightarrow B C_{2} \rightarrow B S O(3)$ shows that $\pi_{1} X \rightarrow \pi_{1} B C_{2}$ is onto and therefore $H^{1}\left(B C_{2}, \mathbb{Z} / 2 \mathbb{Z}\right) \rightarrow H^{1}(X, \mathbb{Z} / 2 \mathbb{Z})$ is an isomorphism so the class of $L$ is nontrivial. Therefore $L \oplus L^{-1}$ is nontrivial by Corollary 2.2 .

We can obtain a 2-dimensional example as follows. The fibration $S O(2) \rightarrow$ $S O(3) \rightarrow S^{2}$ gives us a fibration $S^{1}=S O(2) / C_{2} \rightarrow S O(3) / C_{2} \rightarrow S^{2}$. The homotopy sequence of this shows that $\pi_{1} S^{1} \rightarrow \pi_{1} X$ is onto. Let $n>0$ be divisible by 4 . Since $\pi_{1}(X)=\mathbb{Z} / 4 \mathbb{Z}$, we get a map $Y=S^{1} \cup_{n} e^{2} \rightarrow X$ inducing an epimorphism of $\pi_{1}$. Here $Y$ is obtained by attaching a 2-cell to $S^{1}$ by a map of degree $n$ on the boundary. The composition $Y \rightarrow X \rightarrow B C_{2}$ gives us a 
bundle $L^{\prime}$ on $Y$ which is nontrivial since $\pi_{1} Y \rightarrow \pi_{1} B C_{2}$ is onto and therefore $H^{1}\left(B C_{2}, \mathbb{Z} / 2 \mathbb{Z}\right) \rightarrow H^{1}(Y, \mathbb{Z} / 2 \mathbb{Z})$ is an isomorphism. Since $H^{1}(Y, \mathbb{Z})=0$, it follows from Corollary 2.2 that $L^{\prime} \oplus L^{\prime-1}$ is nontrivial. Of course, $L^{\prime} \oplus L^{\prime-1} \oplus \mathscr{O}$ is trivial since $L^{\prime}$ is induced from $L$.

We have therefore proved the following result (where $\mathscr{O}$ denotes the trivial line bundle).

Theorem 2.3. There exist topological spaces $X$ of the following types with a real line bundle $L$ over $X$ of rank 1 such that $L \oplus L^{-1} \oplus \mathscr{O}$ is trivial but $L \oplus L^{-1}$ is not trivial.

(1) $X$ is a finite 2-dimensional complex.

(2) $X$ is a smooth 3-manifold.

Applying Theorem 6.3 of [SX], we get the following weaker version of Theorem 1(1).

Corollary 2.4. There is a finitely generated $\mathbb{R}$-algebra $R$ of dimension 2 with a finitely generated projective module $L$ over $R$ of rank 1 such that $L \oplus L^{-1} \oplus R$ is free but $L \oplus L^{-1}$ is not free.

\section{COMPLEX BUNDles}

Complex line bundles are classified by maps into $B S^{1}$. As in the real case we consider the fiber $X=S U(3) / S^{1}$ of the map $B S^{1} \rightarrow B S U(3)$ induced by the homomorphism $S^{1} \rightarrow S U(3)$ sending $\alpha$ to the matrix

$$
\left(\begin{array}{ccc}
1 & 0 & 0 \\
0 & \alpha & 0 \\
0 & 0 & \alpha^{-1}
\end{array}\right) \text {. }
$$

Let $L$ be the line bundle on $X$ induced by the map $X \rightarrow B S^{1}$ or, equivalently, the line bundle associated with the principal $S^{1}$-fibration $S U(3) \rightarrow X$. As in the real case, $\mathscr{O} \oplus L \oplus L^{-1}$ is trivial and it only remains to show that $L \oplus L^{-1}$ is nontrivial.

Consider the homomorphism $S^{1} \rightarrow S U(2)$ sending $\alpha$ to the matrix

$$
\left(\begin{array}{cc}
\alpha & 0 \\
0 & \alpha^{-1}
\end{array}\right)
$$

We have an isomorphism $S^{3} \cong S U(2)$ sending a unit quaternion $a+b j$ with $a, b \in \mathbb{C}$ to the matrix

$$
\left(\begin{array}{cc}
a & b \\
-\bar{b} & \bar{a}
\end{array}\right)
$$

Under this isomorphism, the matrix (3.2) corresponds to the quaternion $\alpha$. Therefore $S U(2) / S^{1} \cong S^{3} / S^{1} \cong S^{2}$ by the usual Hopf map $S^{3} \rightarrow S^{2}$. The inclusion $S U(2) \subset S U(3)$ induces an inclusion $S^{2}=S U(2) / S^{1} \subset S U(3) / S^{1}=$ $X$. Now $\pi_{4}(S U(3))=0$ by [SFB, Theorem 25.4] and the homotopy sequence of the fibration $S^{1} \rightarrow S U(3) \rightarrow X$ shows that $\pi_{4}(X)=0$. Since $\pi_{4}\left(S^{2}\right)=\mathbb{Z} / 2 \mathbb{Z}$ the inclusion map $S^{2} \hookrightarrow X$ extends to a map $Y=S^{2} \cup_{\eta} e^{5} \rightarrow X$ where $\eta$ is the generator of $\pi_{4}\left(S^{2}\right)=\mathbb{Z} / 2 \mathbb{Z}$. Let $L^{\prime}$ be the pullback of $L$ to $Y$. It will suffice to show that $L^{\prime} \oplus L^{\prime-1}$ is nontrivial. 
Lemma 3.1. Let $L$ be a complex line bundle on a space $Y$ given by a class $\alpha \in H^{2}(Y, \mathbb{Z})=\left[Y, B S^{1}\right]$. Then $L \oplus L^{-1}$ is trivial if and only if $\alpha$ is spherical. Proof. If $L \oplus L^{-1}$ is trivial, the composition $X \rightarrow B S^{1} \rightarrow B S U(2)$ is homotopic to a constant map so $X \rightarrow B S^{1}$ factors through the fiber $S U(2) / S^{1}=S^{2}$ of the map $B S^{1} \rightarrow B S U(2)$. The converse follows in the same way as in Lemma 2.1. Note that, since $B S U(n)$ is 3-connected, the spectral sequence of the fibration $S U(n) / S^{1} \rightarrow B S^{1} \rightarrow B S U(n)$ shows that $H^{2}\left(B S^{1}, \mathbb{Z}\right) \rightarrow$ $H^{2}\left(S U(n) / S^{1}, \mathbb{Z}\right)$ is an isomorphism. Therefore the maps $S^{2} \hookrightarrow Y \rightarrow X \rightarrow$ $B S^{1}$ all induce isomorphisms of $H^{2}(-, \mathbb{Z})$.

If $L^{\prime} \oplus L^{\prime-1}$ is trivial, the generator of $H^{2}(Y, \mathbb{Z})$ will be spherical so by Lemma 3.2 below, $\pi_{4}\left(S^{2}\right) \rightarrow \pi_{4}(Y)$ will be injective but this is impossible since $\eta$ maps to 0 in $\pi_{4}(Y)$.

Lemma 3.2. Let $Y$ be a topological space and let $f: S^{n} \rightarrow Y$. Suppose there is an element $\alpha \in H^{n}(Y, \mathbb{Z})$ such that $f^{*}(\alpha)=l$ is a generator of $H^{n}\left(S^{n}, \mathbb{Z}\right)$. If $\alpha$ is spherical, then $f$ has a homotopy left inverse.

In other words there is a $g: Y \rightarrow S^{n}$ such that $g f \simeq 1$. This implies that $\pi_{i}\left(S^{n}\right) \rightarrow \pi_{i}(Y)$ will be injective for all $i$.

Proof. We can find a map $g: Y \rightarrow S^{n}$ such that the composition $S^{n} \hookrightarrow Y \rightarrow S^{n}$ induces an isomorphism of $H^{n}(-, \mathbb{Z})$. Therefore it has degree 1 and so is a homotopy equivalence of $S^{n}$ with itself.

We have now proved the following result.

Theorem 3.3. There exist topological spaces $X$ of the following types with a complex line bundle $L$ over $X$ of rank 1 such that $L \oplus L^{-1} \oplus \mathscr{O}$ is trivial but $L \oplus L^{-1}$ is not trivial.

(1) $X$ is a finite 5-dimensional complex.

(2) $X$ is a smooth 7-manifold.

Applying Theorem 6.3 of [SH], we get the following weaker version of Theorem 1 (3).

Corollary 3.4. There is a finitely generated $\mathbb{C}$-algebra $R$ of dimension 5 with $a$ finitely generated projective module $L$ over $R$ with rank 1 such that $L \oplus L^{-1} \oplus R$ is free but $L \oplus L^{-1}$ is not free.

\section{Algebraic examples (ReAl)}

I will now show that the bundles considered in $\S 2$ can be realized over affine subrings of the rings of continuous functions on the spaces considered.

Let $C=\mathbb{R}[x, y] /\left(x^{2}+y^{2}-1\right) \subset C\left(S^{1}\right)$. The map $S^{1} \rightarrow S^{1}$ of degree $n$ given by $z \rightarrow z^{n}$ if $S^{1}=\{z \in \mathbb{C}|| z \mid=1\}$ induces a map $\delta_{n}: C \rightarrow C$ by $\delta_{n}(x)=c_{n}(x, y)$ and $\delta_{n}(y)=s_{n}(x, y)$ where $\cos (n \theta)=c_{n}(\cos \theta, \sin \theta)$ and $\sin (n \theta)=s_{n}(\cos \theta, \sin \theta)$. We have $\mathbb{C} \otimes C=\mathbb{C}[x, y] /\left(x^{2}+y^{2}-1\right)=\mathbb{C}\left[t, t^{-1}\right]$ where $t=x+i y$. If $c$ denotes complex conjugation, $c^{\prime}=c \otimes 1$ acts on $\mathbb{C}\left[t, t^{-1}\right]$ by $c^{\prime}(t)=t^{-1}$. The map $\delta_{n}$ is given by $\delta_{n}(t)=t^{n}$. In particular, $\delta_{n}$ is injective. It also follows that the group of units $\mathrm{U}(C)$ is the subgroup of $\mathrm{U}(\mathbb{C} \otimes C)$ fixed by $c^{\prime}$ which shows that $\mathrm{U}(C)=\mathbb{R}^{*}$. 
Let $\mu_{n}$, the group of $n$th roots of 1 , act on $\mathbb{C} \otimes C=\mathbb{C}\left[t, t^{-1}\right]$ by letting $\zeta \in \mu_{n}$ send $t$ to $\zeta t$. This commutes with the action of $c^{\prime}$ and so induces an action of $\mu_{n}$ on $C$. Since $\mathbb{C}\left[t, t^{-1}\right]^{\mu_{n}}=\mathbb{C}\left[t^{n}, t^{-n}\right]$ and $c^{\prime}\left(t^{n}\right)=t^{-n}$ we see that $C^{\mu_{n}} \approx C$. Moreover, the composition $C \approx C^{\mu_{n}} \hookrightarrow C$ is just $\delta_{n}$ since it sends $t$ to $t^{n}$.

By [SM, Theorem 9.2(a)], we know that $\operatorname{Pic}(C)=\mathbb{Z} / 2 \mathbb{Z}$. I will recall the argument since similar methods will be applied to other cases below.

Definition. If $A \subset B$ is a ring extension, write $P_{n}(B / A)$ for the set of isomorphism classes of finitely generated projective $A$-modules $P$ such that $B \otimes_{A} P \approx$ $B^{n}$.

The familiar galois descent argument [SCL] works without change for galois extensions of rings. A discussion of galois ring extensions can be found in [SN, $\S 2]$.

Theorem 4.1 (galois descent). If $A \subset B$ is a galois extension of rings with galois group $G$, then $P_{n}(B / A) \approx H^{1}\left(G, G L_{n}(B)\right)$.

This is just a reformulation of Speiser's descent theorem [SN, Proposition $2.5]$ since two actions of $G$ on $B^{n}$ differ by a cocycle and isomorphic actions lead to cohomologous cocycles.

Corollary 4.2. If $A \subset B$ is a galois extension of rings with galois group $G$ then $H^{1}\left(G, B^{*}\right)=\operatorname{ker}[\operatorname{Pic}(A) \rightarrow \operatorname{Pic}(B)]$.

By applying this to the galois extension $C \subset \mathbb{C} \otimes C$ and using the fact that $\mathbb{C} \otimes C=\mathbb{C}\left[t, t^{-1}\right]$ has Pic $=0$, we see that $\operatorname{Pic}(C)=H^{1}\left(G, \mathbb{C}\left[t, t^{-1}\right]^{*}\right)=$ $H^{1}\left(G, \mathbb{C}^{*} \times \mathbb{Z}\right)=H^{1}(G, \mathbb{Z})=\mathbb{Z} / 2 \mathbb{Z}$, where $G$ is of order 2 generated by complex conjugation, which acts on $\mathbb{Z}=\langle t\rangle$ by negation. Since the isomorphisms of Theorem 4.1 and Corollary 4.2 are natural, we can compute the effect of $\delta_{n}$ on $\operatorname{Pic}(C)$ by observing that $\delta_{n}$ acts as $n$ on $\mathbb{Z}$, and therefore $\delta_{n}$ also acts by multiplication by $n$ on $\operatorname{Pic}(C)$.

We now consider the space $Z_{n}=S^{1} \cup_{n} e^{2}$ which can be obtained as the pushout of the diagram

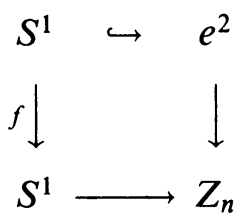

where $f$ is the map of degree $n$ considered above which induces $\delta_{n}: C \rightarrow C$. We always assume that $n>0$. Let $B_{n}$ be the pullback in the diagram

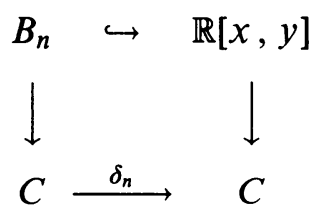

Then $B_{n}$ is a ring of functions on $Z_{n}$. Tensoring (4.1) with $\mathbb{C}$ gives the 
diagram

$$
\begin{array}{ccc}
\mathbb{C} \otimes B_{n} & \hookrightarrow & \mathbb{C}[x, y] \\
\downarrow & & \downarrow \\
\mathbb{C}\left[t, t^{-1}\right] & \stackrel{\delta_{n}}{\longrightarrow} \mathbb{C}\left[t, t^{-1}\right]
\end{array}
$$

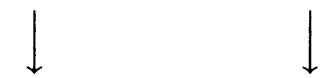

where $\delta_{n}(t)=t^{n}$. Note that $\delta_{n}$ is injective so $B_{n} \hookrightarrow \mathbb{R}[x, y]$.

Lemma 4.3. Let $R$ be the pullback in the diagram

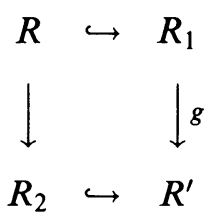

where $g$ is onto. If $R^{\prime}$ is finite over $R_{2}$ then $R_{1}$ is finite over $R$.

Proof. If $S \subset R_{1}$ and $g(S)$ generates $R^{\prime}$ as an $R_{2}$-module, then $S \cup\{1\}$ generates $R_{1}$ as an $R$-module as is easily verified.

This shows that $\mathbb{R}[x, y]$ is finite over $B_{n}$ and hence $B_{n}$ is of finite type over $\mathbb{R}$ by [AT, Theorem 1].

The ( $U$, Pic) Mayer-Vietoris sequence [B, Chapter IX, Theorem 5.3] for (4.1) shows that $0 \rightarrow \operatorname{Pic}\left(B_{n}\right) \rightarrow \operatorname{Pic}(C) \stackrel{n}{\rightarrow} \operatorname{Pic}(C)$ is exact. Since $\operatorname{Pic}(C)=$ $\mathbb{Z} / 2 \mathbb{Z}$, we see that $\operatorname{Pic}\left(B_{n}\right)=\mathbb{Z} / 2 \mathbb{Z}$ for $n$ even and 0 for $n$ odd.

Theorem 4.4. Let $n>0$ be divisible by 4. Let $P$ represent the nontrivial element of $\operatorname{Pic}\left(B_{n}\right)$. Then $P \oplus P^{-1}$ is not free but $P \oplus P^{-1} \oplus B_{n}$ is free.

This proves Theorem $1(1)$. The following lemma and the results of $\S 2$ show that $P \oplus P^{-1}$ is not free.

Lemma 4.5. The map $\operatorname{Pic}\left(B_{n}\right) \rightarrow\left[Z_{n}, B O(1)\right]=H^{1}\left(Z_{n}, \mathbb{Z} / 2 \mathbb{Z}\right)$ is an isomorphism.

Proof. If $n$ is odd both groups are 0. If $n$ is even,

$$
H^{1}\left(Z_{n}, \mathbb{Z} / 2 \mathbb{Z}\right) \rightarrow H\left(S^{1}, \mathbb{Z} / 2 \mathbb{Z}\right)
$$

nd $\operatorname{Pic}\left(B_{n}\right) \rightarrow \operatorname{Pic}(C)$ are isomorphisms. The result follows since $\operatorname{Pic}(C) \rightarrow$ $H^{1}\left(S^{1}, \mathbb{Z} / 2 \mathbb{Z}\right)$ is an isomorphism by [SM, Theorem 7.1].

The fact that $P \oplus P^{-1} \oplus B_{n}$ is free for $n \equiv 0(\bmod 4)$ follows easily from the following result.

Theorem 4.6. $\widetilde{K}_{0}\left(B_{n}\right)=0$ for $n$ odd. If $n \equiv 2(\bmod 4)$, then $\widetilde{K}_{0}\left(B_{n}\right)=\mathbb{Z} / 4 \mathbb{Z}$ generated by $P$, the generator of $\operatorname{Pic}\left(B_{n}\right)$. If $n \equiv 0(\bmod 4)$, then $\widetilde{K}_{0}\left(B_{n}\right)=$ $(\mathbb{Z} / 2 \mathbb{Z}) \oplus(\mathbb{Z} / 2 \mathbb{Z})$.

The proof will be given below. It follows from this that if $n \equiv 0(\bmod 4)$, then $\left[P \oplus P^{-1}\right]=[P \oplus P]=2[P]=2\left[B_{n}\right]$, showing that $P \oplus P^{-1}$ is stably free. Since $\operatorname{dim} B_{n}=2$, Bass' cancellation theorem [B, EE] shows that $P \oplus P^{-1} \oplus B_{n}$ is free as required. Note that if $n \equiv 2(\bmod 4)$, then $2[P]=-2\left[B_{n}\right]$ is not 0 in $K_{0}\left(B_{n}\right)$ so $P \oplus P^{-1}$ is not stably free. 
Lemma 4.7 (Milnor). $S K_{1}(C)=\mathbb{Z} / 2 \mathbb{Z}$, with generator $\eta$ represented by the matrix

$$
\left(\begin{array}{cc}
x & y \\
-y & x
\end{array}\right) \text {. }
$$

See $\left[\mathrm{B}\right.$, p. 714]. Note also that $S K_{1}(\mathbb{C} \otimes C)=S K_{1}\left(\mathbb{C}\left[t, t^{-1}\right]\right)=0$ since $\mathbb{C}\left[t, t^{-1}\right]$ is Euclidean.

Corollary 4.8. The transfer map $U(\mathbb{C} \otimes C)=K_{1}(\mathbb{C} \otimes C) \rightarrow K_{1}(C)$ sends $t$ to $\eta$.

Proof. One easily checks that under the isomorphism $\mathbb{C} \otimes C \approx C^{2}$, multiplication by $t$ corresponds to the matrix of Lemma 4.7.

Corollary 4.9. The map $S K_{1}(C) \rightarrow S K_{1}(C)$ induced by $\delta_{n}$ is multiplication by $n$.

This is clear from Corollary 4.8 since $\delta_{n}$ sends $t$.to $t^{n}$.

Proof of Theorem 4.6. Using Corollary 4.9 and the similar result for Pic proved above following Corollary 4.2 , the $\left(K_{1}, K_{0}\right)$ Mayer-Vietoris sequence [B, Chapter IX, Theorem 5.3] for (4.1) shows that $\widetilde{K}_{0}\left(B_{n}\right)=0$ for $n$ odd while, for $n$ even, we have an exact sequence

$$
0 \rightarrow S K_{1}(C) \rightarrow \widetilde{K}_{0}\left(B_{n}\right) \rightarrow \operatorname{Pic}\left(B_{n}\right) \rightarrow 0,
$$

which is isomorphic to

$$
0 \rightarrow \mathbb{Z} / 2 \mathbb{Z} \rightarrow \widetilde{K}_{0}\left(B_{n}\right) \rightarrow \mathbb{Z} / 2 \mathbb{Z} \rightarrow 0 .
$$

The same sequence for (4.2) shows that for all $n, \widetilde{K}_{0}\left(\mathbb{C} \otimes B_{n}\right)=\operatorname{Pic}\left(\mathbb{C} \otimes B_{n}\right)=$ $\mathbb{Z} / n \mathbb{Z}$ generated by the image of $t \in K_{1}(\mathbb{C} \otimes C)=U(\mathbb{C} \otimes C)$. Assume that $n$ is even. It is clear from the construction of the boundary map in the MayerVietoris sequence that the diagram

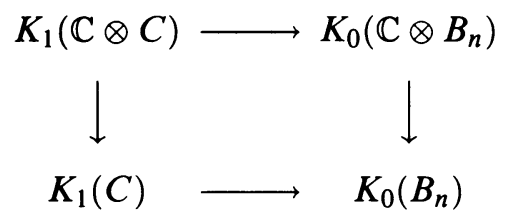

is commutative. By Corollary 4.8 , we see that the generator of $\widetilde{K}_{0}\left(\mathbb{C} \otimes B_{n}\right)=$ $\mathbb{Z} / n \mathbb{Z}$ maps to the generator of the image $\mathbb{Z} / 2 \mathbb{Z}$ of $S K_{1}(C)$ in $\widetilde{K}_{0}\left(B_{n}\right)$. Consider the composition

$$
\operatorname{Pic}\left(B_{n}\right) \rightarrow \operatorname{Pic}\left(\mathbb{C} \otimes B_{n}\right)=\widetilde{K}_{0}\left(\mathbb{C} \otimes B_{n}\right) \rightarrow \widetilde{K}_{0}\left(B_{n}\right)
$$

which takes the form

$$
\mathbb{Z} / 2 \mathbb{Z} \rightarrow \mathbb{Z} / n \mathbb{Z} \rightarrow \mathbb{Z} / 2 \mathbb{Z}=S K_{0}\left(B_{n}\right),
$$

and sends $P \in \operatorname{Pic}\left(B_{n}\right)$ to $2[P]-2\left[B_{n}\right]$ in $S K_{0}\left(B_{n}\right) \subset \widetilde{K}_{0}\left(B_{n}\right)$.

If $n \equiv 0(\bmod 4)$, this composition is necessarily 0 showing that $2[P]-$ $2\left[B_{n}\right]=0$. Since $[P]-\left[B_{n}\right]$ maps to $P$ in $\operatorname{Pic}\left(B_{n}\right)$, the sequence (4.3) splits and $\widetilde{K}_{0}\left(B_{n}\right) \approx \mathbb{Z} / 2 \mathbb{Z} \oplus \mathbb{Z} / 2 \mathbb{Z}$.

Now $\operatorname{Pic}\left(B_{n}\right) \rightarrow \operatorname{Pic}\left(\mathbb{C} \otimes B_{n}\right)$ is injective since, by Corollary 4.2, its kernel is $H^{1}\left(G,\left(\mathbb{C} \otimes B_{n}\right)^{*}\right)=H^{1}\left(G, \mathbb{C}^{*}\right)=0$. Therefore if $n \equiv 2(\bmod 4)$, the 
composition (4.4) is an isomorphism on the 2-components. This shows that $2[P]-2\left[B_{n}\right]$ generates $S K_{0}\left(B_{n}\right)$ and hence $[P]-\left[B_{n}\right]$ generates $\widetilde{K}_{0}\left(B_{n}\right)$ so $\widetilde{K}_{0}\left(B_{n}\right)=\mathbb{Z} / 4 \mathbb{Z}$.

To prove (2) of Theorem 1, we will consider the coordinate ring of $S O(3) / C_{2}$ $=S^{3} / C_{4}$. The group $C_{4}=\langle i\rangle$ acts freely on $S^{3}$ and even on its complex points, i.e. the complex solutions of $x_{0}^{2}+\cdots+x_{3}^{2}=1$. This is clear since no point $x$ of $S^{3}$ has $x=-x$. Recall the following standard fact.

Theorem 4.10. Let $X=\operatorname{Spec} A$ where $A$ is an affine algebra over a field $k$. Let $G$ be a finite group of automorphisms of $A$ as a $k$-algebra. Assume that $G$ acts freely on $X(\bar{k})$ where $\bar{k}$ is the algebraic closure of $k$. Then $A$ is a galois ring extension of the fixed subalgebra $A^{G}$ with galois group $G$. In particular, $A$ is finite and étale over $A^{G}$ so $A^{G}$ is smooth over $k$ if and only if $A$ is.

Proof. The extension $A / A^{G}$ is galois with group $G$ if and only if the following condition holds (see [SN, §2] and references given there).

For all subgroups $H$ of $G$ and all $H$-stable proper ideals $I$ of $A, H$ acts faithfully on $A / I$.

Suppose this condition is not satisfied. Let $Y=\operatorname{Spec} A / I \subset X$. If $\sigma \neq 1$ in $H$ acts trivially on $A / I$, then $\sigma$ acts trivially on $Y(\bar{k})$. This contradicts the hypothesis since $Y(\bar{k})$ is nonempty by Hilbert's Nullstellensatz.

It is well known that galois extensions are étale. In fact, it is enough to check this after making a faithfully flat extension of $A^{G}[\mathrm{R}$, Chapter II, Proposition 4]. By [SN, §2] we can find such an extension splitting $A$ over $A^{G}$. Finally, $A^{G}$ is smooth if and only if $A$ is, since smoothness is a local property in the étale topology ([SGA1, Chapter I, Corollary 9.2] and [SGA1, Chapter II, Theorem $5.5 \mathrm{i}, \mathrm{iv}])$.

Now let $A=\mathbb{R}\left[x_{0}, \ldots, x_{3}\right] /\left(x_{0}^{2}+\cdots+x_{3}^{2}-1\right)$ be the coordinate ring of $S^{3}$. Let $G=\left\langle\sigma: \sigma^{4}=1\right\rangle$ acting on $A$ by $\sigma\left(x_{0}, x_{1}, x_{2}, x_{3}\right)=\left(-x_{1}, x_{0},-x_{3}, x_{2}\right)$. This corresponds to left multiplication by $i$ on quaternions $q=x_{0}+x_{1} i+$ $x_{2} j+x_{3} k$. Let $R=A^{G}$. Since $\operatorname{Pic}(A)=0$ [SM, Theorem 9.2], Corollary 4.2 shows that $\operatorname{Pic}(R)=H^{1}\left(G, A^{*}\right)=H^{1}\left(G, \mathbb{R}^{*}\right)=\operatorname{Hom}\left(G, \mathbb{R}^{*}\right)=\{ \pm 1\}$.

Theorem 4.11. Let $P$ represent the nontrivial element of $\operatorname{Pic}(R)$. Then $P \oplus P^{-1}$ is not free but $P \oplus P^{-1} \oplus R$ is free.

This, together with Lemma 4.13 below, proves (2) of Theorem 1.

Proof. The module $R \oplus P \oplus P^{-1}$ corresponds to the cocycle sending $\sigma$ to the matrix

$$
J=\left(\begin{array}{ccc}
1 & 0 & 0 \\
0 & -1 & 0 \\
0 & 0 & -1
\end{array}\right)
$$

in $G L_{3}(A)$. We will show that it is a coboundary.

Let $\Lambda=A \otimes_{\mathbb{R}} \mathbb{H}$. We write the elements of $\Lambda$ as $a=a_{0}+a_{1} i+a_{2} j+a_{3} k$ with $a_{i} \in A$. Let $\bar{a}=a_{0}-a_{1} i-a_{2} j-a_{3} k$ and set $\mathscr{R} a=a_{0}$. Note that $a \bar{a}=\sum a_{i}^{2}$. Define $(a, b)=\mathscr{R} a \bar{b}$. If $b=b_{0}+b_{1} i+b_{2} j+b_{3} k$ then $(a, b)=\sum a_{i} b_{i}=(\bar{a}, \bar{b})$. We have $(a c, b c)=c \bar{c}(a, b)=(c a, c b)$. 
Generalizing the classical map $\varphi: S^{3} \rightarrow S O(3)$, we define $\varphi: U(\Lambda) \rightarrow G L_{3}(A)$ by sending $q$ to the map $a \mapsto q a q^{-1}$ of $A i+A j+A k$ to itself. This space consists of the elements $a$ with $(a, 1)=0$ and so is stable under conjugation by $q$. Clearly $\varphi$ commutes with automorphisms of $A$ such as $\sigma$. Choose $q=x_{0}+x_{1} i+x_{2} j+x_{3} k$. Then $q \bar{q}=1$ so $q$ lies in $U(\Lambda)$. Since $\sigma(q)=i q$ by the definition of $\sigma$ we have $\sigma(\varphi(q)) \varphi(q)^{-1}=\varphi(i q) \varphi(q)^{-1}=\varphi(i)=J$ as required. Therefore $J$ is a coboundary so $P \oplus P^{-1} \oplus R$ is free.

Remark. Explicitly,

$$
\varphi(q)=\left(\begin{array}{ccc}
x_{0}^{2}+x_{1}^{2}-x_{2}^{2}-x_{3}^{2} & 2\left(x_{1} x_{2}-x_{0} x_{3}\right) & 2\left(x_{0} x_{2}+x_{1} x_{3}\right) \\
2\left(x_{0} x_{3}+x_{1} x_{2}\right) & x_{0}^{2}-x_{1}^{2}+x_{2}^{2}-x_{3}^{2} & 2\left(x_{2} x_{3}-x_{0} x_{1}\right) \\
2\left(x_{1} x_{3}-x_{0} x_{2}\right) & 2\left(x_{0} x_{1}+x_{2} x_{3}\right) & x_{0}^{2}-x_{1}^{2}-x_{2}^{2}+x_{3}^{2}
\end{array}\right) .
$$

Using this it is easy to verify directly that $J=\sigma(\varphi(q)) \varphi(q)^{-1}$. Also, if $D$ is the coordinate ring of $S O(3)$, the map $D \rightarrow A^{C_{2}}=A_{\text {even }}$ induced by $\varphi$ is easily checked to be onto and therefore an isomorphism since both domains have dimension 3.

Clearly $R=A^{G}$ can be regarded as a ring of functions on $X=S O(3) / C_{2}=$ $S^{3} / G$. We will show that $P \oplus P^{-1}$ is not free by using the results of $\S 2$.

Lemma 4.12. $P$ induces the bundle $L$ on $X$.

Proof. Consider the inclusion $S^{1}=S O(2) / C_{2} \hookrightarrow X=S O(3) / C_{2}$. Since $H^{1}(X, \mathbb{Z} / 2 \mathbb{Z}) \rightarrow H^{1}\left(S^{1}, \mathbb{Z} / 2 \mathbb{Z}\right)=\mathbb{Z} / 2 \mathbb{Z}$ is an isomorphism, it is enough to show that $P$ induces the Möbius bundle on $S^{1}$.

The restriction map $C\left(S^{3}\right) \hookrightarrow C\left(S^{1}\right)$ induces the homomorphism $A \rightarrow C$ sending $x_{0}$ to $x, x_{1}$ to $y$, and $x_{2}$ and $x_{3}$ to 0 . This in turn induces a map $A^{G} \rightarrow C^{G}$ corresponding to $S^{1}=S^{1} / G \hookrightarrow X=S^{3} / G$ and the results at the beginning of this section show that $C^{G}$ is just the coordinate ring of $S^{1}=$ $S^{1} / G$. In particular, the nontrivial element of $\operatorname{Pic}\left(C^{G}\right)$ induces the Möbius bundle on $S^{1}$. The map $H^{1}\left(G, A^{*}\right) \rightarrow H^{1}\left(G, C^{*}\right)$ is an isomorphism since $A^{*}=C^{*}=\{ \pm 1\}$. Therefore $\operatorname{Pic}\left(A^{G}\right) \rightarrow \operatorname{Pic}\left(C^{G}\right)$ is nontrivial and hence an isomorphism, both groups being $\mathbb{Z} / 2 \mathbb{Z}$.

Lemma 4.13. $R=A^{G}$ is rational over $\mathbb{R}$.

Proof. Recall that $A=\mathbb{R}\left[x_{0}, \ldots, x_{3}\right] /\left(x_{0}^{2}+\cdots+x_{3}^{2}-1\right)$ with $G=\left\langle\sigma: \sigma^{4}=1\right\rangle$ acting on $A$ by $\sigma\left(x_{0}, x_{1}, x_{2}, x_{3}\right)=\left(-x_{1}, x_{0},-x_{3}, x_{2}\right)$. Therefore $\sigma^{2}$ acts by sending $x_{i}$ to $-x_{i}$ for all $i$. Let $K$ be the quotient field of $A$. Then $K=\mathbb{R}\left(\xi_{1}, \xi_{2}, \xi_{3}, \rho\right)$ where $\xi_{i}=x_{i} / x_{0}$ and $\rho=1 / x_{0}$. Here $\xi_{1}, \xi_{2}$, and $\xi_{3}$, are fixed by $\sigma^{2}$ while $\rho$ is sent to $-\rho$. Since $\rho^{2}=1+\xi_{1}^{2}+\xi_{2}^{2}+\xi_{3}^{2}$, it is clear that the subfield $K$ fixed by $\sigma^{2}$ is $L=\mathbb{R}\left(\xi_{1}, \xi_{2}, \xi_{3}\right)$. Now $\alpha=\xi_{2} \xi_{3} / \xi_{1}$ is fixed by $\sigma$. If we let $F=\mathbb{R}(\alpha)$, then $L=F\left(\xi_{1}, \xi_{2}\right)$ and $\sigma$ acts by $\sigma\left(\xi_{1}\right)=-\xi_{1}^{-1}$ and $\sigma\left(\xi_{2}\right)=\alpha \xi_{2}^{-1}$. Since $\sigma$ acts monomially, it follows from a theorem of Hajja [H, Theorem 4] that $L$ is rational over $F$ and hence over $\mathbb{R}$.

\section{KNOWN RESULTS}

For the reader's convenience, I will recall here some standard results on the special linear group, complex spheres, and $\mathbb{G}_{m}$-bundles. 
The real coordinate ring $A_{R}$ of $S U(n)$ has generators $x_{i j}$ and $y_{i j}$ with relations expressing the fact that the matrix $Z=\left(z_{\mu \nu}\right)$ with $z_{\mu \nu}=x_{\mu \nu}+i y_{\mu \nu}$ satisfies the relations $\bar{Z}^{T} Z=I$ and $\operatorname{det}(Z)=1$.

Let $A$ be the coordinate ring of $S L_{3}$ over $\mathbb{C}$.

Lemma 5.1. $\mathbb{C} \otimes_{\mathrm{R}} A_{R} \stackrel{\approx}{\longrightarrow} A$.

Proof. Over $\mathbb{C}$, we can change the variables from $x_{i j}$ and $y_{i j}$ to $z_{i j}$ and $\bar{z}_{i j}$ where $\bar{z}_{i j}=x_{i j}-i y_{i j}$. Since $\bar{Z}^{T} Z=I$, the variables $\bar{z}_{i j}$ are superfluous and, after eliminating them, the only remaining relation is $\operatorname{det}(Z)=1$.

Lemma 5.2. $A$ is a smooth rational domain of dimension $n^{2}-1$.

Proof. If $m_{\mu \nu}$ is the cofactor of $z_{\mu \nu}$ in the matrix $Z=\left(z_{\mu \nu}\right)$, then $A\left[m_{\mu \nu}^{-1}\right]$ is clearly a localization of the polynomial ring of the $n^{2}-1$ variables $z_{\alpha \beta}$ with $(\alpha, \beta) \neq(\mu, \nu)$. Since the minors corresponding to any row span the unit ideal, it follows that $A$ is smooth. Since we can find matrices with all minors nonzero, the corresponding affine pieces of $S L_{n}(\mathbb{C})$ overlap nontrivially showing that $S L_{n}(\mathbb{C})$ is irreducible, so $A$ is a domain.

If $B$ is a finitely generated $\mathbb{R}$-algebra, let $V_{R}(B)$ be the set of $\mathbb{R}$-algebra homomorphisms $f: B \rightarrow \mathbb{R}$. Let $b_{1}, \ldots, b_{n}$ generate $B$ and embed $V_{R}(B)$ in $\mathbb{R}^{n}$ by sending $f$ to $\left(f\left(b_{1}\right), \ldots, f\left(b_{n}\right)\right)$.

Lemma 5.3. $\operatorname{dim} V_{R}(B) \leq \operatorname{dim} B$.

Proof. By Noether's normalization lemma, $B$ is integral over a polynomial ring $C=\mathbb{R}\left[x_{1}, \ldots, x_{d}\right]$ where $d=\operatorname{dim} B$. The inclusion $C \subset B$ induces a map $V_{R}(B) \rightarrow V_{R}(C)=\mathbb{R}^{d}$ with finite fibers and the result follows.

Let $C_{R}(X)$ denote the ring of continuous real functions on $X$.

Lemma 5.4. $A_{R} \rightarrow C_{R}(S U(n))$ is injective.

Tensoring with $\mathbb{C}$ shows that $A \rightarrow C_{C}(S U(n))$ is also injective.

Proof. Let $B$ be the image of $A_{R} \rightarrow C_{R}(S U(n))$. If this map is not injective, $B$ is a proper quotient of $A$ and so $\operatorname{dim} B<n^{2}-1$. Since $V_{R}(B)=S U(n)$ has dimension $n^{2}-1$, this contradicts Lemma 5.3.

Define the "complex $n$-sphere" $S C^{n}$ to be

$$
S C^{n}=\left\{\left(z_{0}, \ldots, z_{n}\right) \in \mathbb{C}^{n+1} \mid z_{0}^{2}+\cdots+z_{n}^{2}=1\right\} .
$$

The following fact is presumably also well known.

Lemma 5.5. $S C^{n}$ is diffeomorphic to the tangent bundle $T\left(S^{n}\right)$ of $S^{n}$.

Proof. Let $z_{\nu}=x_{\nu}+i y_{\nu}$ with $x_{\nu}$ and $y_{\nu}$ in $\mathbb{R}^{n+1}$. Then $S C^{n}$ is defined by the equations $\sum x_{\nu}^{2}-\sum y_{\nu}^{2}=1$ and $\sum x_{\nu} y_{\nu}=0$. On the other hand, $T\left(S^{n}\right)$ is the set of $(t, y)$ in $\mathbb{R}^{n+1} \times \mathbb{R}^{n+1}$ satisfying $\sum t_{\nu}^{2}=1$ and $\sum t_{\nu} y_{\nu}=0$. The required diffeomorphism is given by $t=x /|x|$ and $x=\left(1+\sum y_{\nu}^{2}\right)^{1 / 2} t$.

Corollary 5.6. The inclusion $S^{n} \subset S C^{n}$ is a homotopy equivalence.

Remark. The tangent bundle $T\left(S^{2}\right)$ has another algebraic structure obtained by identifying $S^{2}$ with the complex projective line $\mathbb{P}_{\mathbb{C}}^{1}$. In contrast to $S C^{2}$, this structure is not affine since the zero section is a subvariety isomorphic to 
$\mathbb{P}^{1}$. Thus $T\left(S^{2}\right)$ is a simple example of a real 4-manifold with two distinct structures as a complex variety.

We now recall some standard results on $\mathbb{G}_{m}$-actions as motivation for the construction given in $\S 6$. If $\mathbb{G}_{m}=\operatorname{Spec} \mathbb{Z}\left[t, t^{-1}\right]$ acts on an affine scheme $X=\operatorname{Spec} A$, the map $\mathbb{G}_{m} \times X \rightarrow X$ corresponds to a map $\varphi: A \rightarrow A\left[t, t^{-1}\right]$. Write $\varphi(a)=\sum e_{n}(a) t^{n}$ where $e_{n}: A \rightarrow A$. For each $a, e_{n}(a)=0$ except for a finite number of $n$. The relation $1 \cdot x=x$ translates into $\sum e_{n}(a)=a$, and the relation $\sigma(\tau a)=(\sigma \tau) a$ is easily checked to be equivalent to $e_{m}\left(e_{n}(a)\right)=0$ for $m \neq n$, and $e_{n}\left(e_{n}(a)\right)=e_{n}(a)$. Therefore the $e_{n}$ are orthogonal idempotents so $A=\bigoplus A_{n}$, where $A_{n}=\operatorname{im} e_{n}$. Also, the condition that $\varphi$ be a ring homomorphism is equivalent to $A_{p} \cdot A_{q} \subset A_{p+q}$. This shows that a $\mathbb{G}_{m}$-action on $\operatorname{Spec} A$ is equivalent to a $\mathbb{Z}$-granding of $A$. If $f: \operatorname{Spec} A \rightarrow \operatorname{Spec} B$ is a principal $\mathbb{G}_{m}$-bundle, $A$ must look locally like $B\left[t, t^{-1}\right]$ with the obvious grading. It follows that $B=A_{0}$. In particular, we see that $\operatorname{Spec} A$ is smooth if and only if $\operatorname{Spec} B$ is. It is enough to check this locally and it is clear that $B\left[t, t^{-1}\right]$ is smooth if and only if $B$ is.

It is easy to determine the required grading in a specific example since if $\alpha \in \mathbb{G}_{m}(\mathbb{C})$ then $\alpha: \operatorname{Spec} A \rightarrow \operatorname{Spec} A$ induces the map $A \rightarrow A$ sending $\sum a_{n}$ to $\sum a_{n} \alpha^{n}$.

\section{Algebraic eXamples (COMPlex)}

We first construct a coordinate ring for $X=S U(3) / S^{1}$ where $S^{1}$ consists of the matrices

$$
\left(\begin{array}{ccc}
1 & 0 & 0 \\
0 & \alpha & 0 \\
0 & 0 & \alpha^{-1}
\end{array}\right)
$$

with $\alpha \in \mathbb{C},|\alpha|=1$.

By analogy with Lemma 5.1, we will do this by working with $S L_{3}(\mathbb{C})$ instead. Let $T$ be the subgroup of $S L_{3}(\mathbb{C})$ consisting of all matrices (6.1) with $\alpha \in \mathbb{C}^{*}$, and let $X^{\prime}=S L_{3}(\mathbb{C}) / T$. Note that $X=S U(3) / S^{1} \rightarrow X^{\prime}$ is a homotopy equivalence since $S U(3) \rightarrow S L_{3}(\mathbb{C})$ and $S^{1} \rightarrow \mathbb{C}^{*}$ are, and considering the homotopy sequences of the fibrations

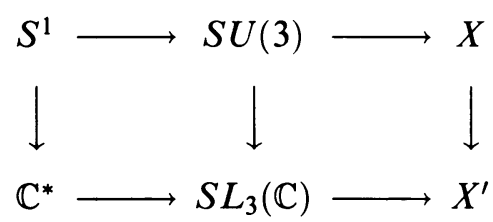

shows that $X \rightarrow X^{\prime}$ induces an isomorphism of homotopy groups.

Now let $A$ be the coordinate ring of $S L_{3}$ over $\mathbb{C}$, and let $\mathbb{G}_{m}(\mathbb{C}) \cong T$ act by right multiplication on $S L_{3}$. Following the discussion at the end of $\S 5$, we define a grading $A=\bigoplus A_{n}$ on $A$ by letting $\operatorname{deg} z_{i j}=0$ if $j=1,1$ if $j=2$, and -1 if $j=3$. Let $\bar{Z}^{T}$ be the inverse of $Z$ and define $\operatorname{det} \bar{z}_{i j}=-\operatorname{deg} z_{i j}$ making $\bar{Z}^{T} Z=I$ a homogeneous equation. If $S$ is the matrix given by (6.1) then the mapping $Z \rightarrow Z S$ corresponds to the automorphism of $A$ which is induced by multiplication by $\alpha^{n}$ on $A_{n}$. Let $B=A_{0}$, the set of elements of degree 0 . Then $B$ is the set of elements of $A$ invariant under the action of $S^{1}$ 
so $B$ can be regarded as a ring of functions on $X=S U(3) / S^{1}$ as well as the coordinate ring of $X^{\prime}=S L_{3}(\mathbb{C}) / T$.

Lemma 6.1. $A$ is locally (on $\operatorname{Spec} B$ ) isomorphic to $B\left[t, t^{-1}\right]$.

Proof. We have $\sum \bar{z}_{j 2} z_{j 2}=1$ so, if $a_{j}=\bar{z}_{j 2} z_{j 2}$, it is enough to prove that $A\left[a_{j}^{-1}\right]=B\left[a_{j}^{-1}\right]\left[t, t^{-1}\right]$ for some $t$. But this is true with $t=z_{j 2}$ since this is a unit of degree 1 in $A\left[a_{j}^{-1}\right]$.

Lemma 6.2. $B$ is a smooth rational $\mathbb{C}$-algebra.

Proof. $B$ is smooth by Lemma 5.2 and the remarks at the end of $\S 5$. Referring to the proof of Lemma 6.1 , we see that it will suffice to show that $B\left[a_{1}^{-1}\right]$ is rational. This is a localization of $A /\left(z_{12}-1\right)$ which is the coordinate ring of the subvariety $W$ of $S L_{3}(\mathbb{C})$ consisting of matrices with $z_{12}=1$. Such matrices can be reduced to the form $\left(\begin{array}{ll}1 & 0 \\ 0 & M\end{array}\right)$ with $M \in S L_{2}(\mathbb{C})$ by a canonically chosen series of elementary transformations. This shows that $W \cong \mathbb{C}^{4} \times S L_{2}(\mathbb{C})$ showing that $W$ is rational by Lemma 5.2.

We choose $P=A_{-1}$, which is a finitely generated projective $B$-module of rank 1 with $P^{-1} \approx A_{1}$. This follows immediately from Lemma 6.1 since the analogous statement for $B\left[t, t^{-1}\right]$ is trivial.

Theorem 6.3. $P \oplus P^{-1}$ is not free but $P \oplus P^{-1} \oplus B$ is free.

Proof. Let $\varphi: A \rightarrow A$ be defined by $(a, b, c) \rightarrow(a, b, c) Z=(a, b, c)\left(z_{i j}\right)$ (matrix product). This is an automorphism with inverse $\varphi^{-1}:(a, b, c) \rightarrow$ $(a, b, c) \bar{Z}^{T}$. Clearly $\varphi$ sends $A_{0} \oplus A_{0} \oplus A_{0}$ to $A_{0} \oplus A_{1} \oplus A_{-1}$ while $\varphi^{-1}$ sends $A_{0} \oplus A_{1} \oplus A_{-1}$ to $A_{0} \oplus A_{0} \oplus A_{0}$. Therefore $\varphi: B^{3}=A_{0}^{3} \approx A_{0} \oplus A_{1} \oplus A_{-1} \approx$ $B \oplus P^{-1} \oplus P$. The fact that $P \oplus P^{-1}$ is not free follows from the next lemma and the results of $\S 3$.

Lemma 6.4. $P$ induces the line bundle $L$ of $\S 3$ on $X$.

Proof. The line bundle $L$ is the complex vector bundle associated to the principal bundle $S^{1} \rightarrow S U(3) \rightarrow X$. The map $S^{1} \rightarrow S U(3)$ sends $\alpha \in S^{1}$ to the matrix $S$ given in (6.1). Therefore, $L$ is the quotient $(S U(3) \times \mathbb{C}) / \sim$ where $(Z, \alpha \zeta) \sim(Z S, \zeta)$ for $\alpha \in S^{1}$. Let $Z^{\prime}$ denote the image in $X$ of $Z \in S U(3)$. A section of $L$ is given by $Z^{\prime} \rightarrow(Z, \varphi(Z))$ where $(Z S, \varphi(Z S))=(Z, \varphi(Z))$ for $\alpha \in S^{1}$ or, equivalently, $\varphi(Z S)=\alpha^{-1} \varphi(Z)$. If $\varphi$ belongs to the ring $A$, this is exactly the condition that $\varphi=A_{-1}=P$. Therefore we have a map $P \rightarrow \Gamma(L)$ which is clearly a $B$-homomorphism and so induces a map $C(X) \otimes_{B} P \rightarrow \Gamma(L)$. This corresponds to a map of line bundles $E \rightarrow L$ where $E$ is the bundle induced by $P$. To see that $E \approx L$ it is enough to check that $E_{x} \rightarrow L_{x}$ is nonzero at all points $x$ of $X$. This is clear since the $\bar{z}_{i 2}$ lie in $A_{-1}=P$ and one will be nonzero at $x$.

To get a 5 dimensional example as required by Theorem 1 (3) we can consider the space $S^{2} \cup_{\eta} e^{5}$ as in $\S 3$, but a slight modification of this will be more convenient.

In the following lemma $a, \bar{a}, b, \bar{b}, c, \bar{c}$ can be any elements satisfying the given relation (not necessarily complex conjugates). 
Lemma 6.5. If $a \bar{a}+b \bar{b}+c \bar{c}=1$, then the matrix

$$
\left(\begin{array}{ccc}
1-(a+\bar{a}) \bar{a} & (a+\bar{a}) b & (a+\bar{a}) c \\
\bar{a} b+i \bar{c} & -b^{2}-\bar{c}^{2}-i a b \bar{c} & -b c+\overline{b c}+i a+i a b \bar{b} \\
\bar{a} c-i \bar{b} & -b c+\overline{b c}-i a-i a c \bar{c} & -c^{2}-\bar{b}^{2}+i a \bar{b} c
\end{array}\right)
$$

has determinant 1 .

Now let $S^{5} \subset \mathbb{C}^{3}$ with coordinates $(a, b, c)$ and let $\bar{a}, \bar{b}, \bar{c}$ be the complex conjugates of $a, b, c$. Let $\varphi: S^{5} \rightarrow S L_{3}(\mathbb{C})$ by sending $(a, b, c)$ to the matrix obtained from that of Lemma 6.5 by the following elementary transformations

$$
\left(\begin{array}{l}
\operatorname{Row}_{1} \\
\operatorname{Row}_{2}-(\bar{a} b+i \bar{c}) \text { Row }_{1} \\
\operatorname{Row}_{3}-(\bar{a} c-i \bar{b}) \text { Row }_{1}
\end{array}\right) .
$$

These elementary transformations were chosen so that the equator $S^{4}$ of $S^{5}$ defined by $a+\bar{a}=0$ is mapped into $S L_{2}(\mathbb{C})$ (the subgroup with first row and column $(100)$ ) by $\varphi$. Let $\pi: S L_{3}(\mathbb{C}) \rightarrow \mathbb{C}^{3}-\{0\}$ by sending $\sigma$ to its first row $p \sigma$ where $p=(1,0,0)$. Then $\pi \varphi$ sends $S^{4}$ to $p$ while the north and south poles $( \pm 1,0,0)$ are sent to $(-1,0,0)$.

Lemma 6.6. $\pi \varphi$ maps $S^{5}$ onto $S^{5} \subset \mathbb{C}^{3}$ and each hemisphere maps onto $S^{5}$ with degree 1.

Proof. In real coordinates $\left(x_{0}, x_{1}, \ldots, x_{5}\right)$ with $a=x_{0}+i x_{1}$, etc., $\pi \varphi$ is given by $\pi \varphi\left(x_{0}, x_{1}, \ldots, x_{5}\right)=\left(1-2 x_{0}^{2}, 2 x_{0} x_{1}, \ldots, 2 x_{0} x_{5}\right)$ which clearly has the described properties.

This map can be described geometrically as follows. Pinch the equator $S^{4}$ to a point getting two spheres with a common point. Then fold these two spheres into a single sphere, identifying the images of the north and south poles of the original sphere.

As observed above, the restriction of $\varphi$ to $S^{4}$ takes the form

$$
\varphi(x)=\left(\begin{array}{cc}
1 & 0 \\
0 & \psi(x)
\end{array}\right)
$$

where, if $a=i r$ with $r \in \mathbb{R}$,

$$
\psi(X)=\left(\begin{array}{cc}
-b^{2}-\bar{c}^{2}+r b \bar{c} & -b c+\overline{b c}-r-r b \bar{b} \\
-b c+\overline{b c}+r+r c \bar{c} & -c^{2}-\bar{b}^{2}-r \bar{b} c
\end{array}\right) .
$$

If we let $\varphi^{+}$be the restriction of $\varphi$ to the upper hemisphere of $S^{5}$, then $\varphi^{+}: E^{5} \rightarrow S L_{3}(\mathbb{C})$ lifts the map $E^{5} \rightarrow E^{5} / S^{4} \stackrel{\cong}{\longrightarrow} S^{5}$. Therefore $\varphi^{+} \mid S^{4}=\psi$ represents the image of the generator of $\pi_{5}\left(S^{5}\right)=\pi_{5}\left(\mathbb{C}^{3}-\{0\}\right)$ under the map $\partial$ in the homotopy sequence

$$
\pi_{5}\left(\mathbb{C}^{3}-\{0\}\right) \stackrel{\partial}{\longrightarrow} \pi_{4}(H) \rightarrow \pi_{4}\left(S L_{3}(\mathbb{C})\right) \rightarrow \pi_{4}\left(\mathbb{C}^{3}-\{0\}\right)
$$

of $H \rightarrow S L_{3}(\mathbb{C}) \rightarrow \mathbb{C}^{3}-\{0\}$. Here $H$ is the group of matrices in $S L_{3}(\mathbb{C})$ of the form

$$
\left(\begin{array}{lll}
1 & 0 & 0 \\
* & * & * \\
* & * & *
\end{array}\right)
$$


This clearly has the same homotopy type as $S L_{2}(\mathbb{C})$, embedded in $S L_{3}(\mathbb{C})$ as

$$
\left(\begin{array}{lll}
1 & 0 & 0 \\
0 & * & * \\
0 & * & *
\end{array}\right) .
$$

Since $\pi_{4}\left(S L_{3}(\mathbb{C})\right)=\pi_{4}(S U(3))=\pi_{4}(S U)=0$ and $\pi_{4}(H)=\pi_{4}\left(S L_{2}(\mathbb{C})\right)=$ $\pi_{4}(S U(2))=\pi_{4}\left(S^{3}\right)=\mathbb{Z} / 2 \mathbb{Z}$, we see that $\psi$ represents the generator of $\pi_{4}(H)$.

Now consider the compositions

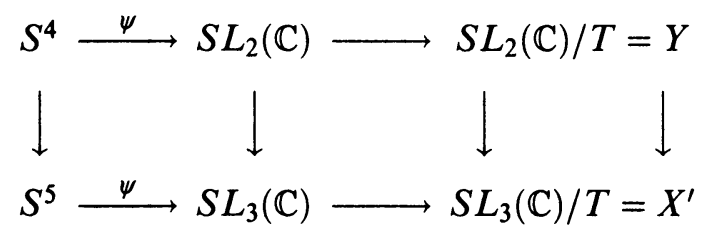

We can find the coordinate ring of $Y$ in the same way as that of $X^{\prime}$ above. If $\left(\begin{array}{ll}a & b \\ c & d\end{array}\right)$ is a generic matrix in $S L_{2}(\mathbb{C})$, we grade $\mathbb{C}[a, b, c, d] /(a d-b c-1)$, the coordinate ring of $S L_{2}(\mathbb{C})$, by letting $a$ and $c$ have degree 1 and letting $b$ and $d$ have degree -1 . The subring of elements of degree 0 then has generators $p=a b, q=a d, r=c b, s=c d$ with relations $q-r=1$ and $p s=q r$. Eliminating $q$, this reduces to $p s=r(r+1)$ which can be transformed to the form $t_{0}^{2}+t_{1}^{2}+t_{2}^{2}=1$ by setting $t_{0}=q+r, t_{1}=p-s, t_{2}=i(p+s)$. Therefore $Y$ is the complex sphere $S C^{2}$ discussed in $\S 5$. The map $S L_{2}(\mathbb{C}) \rightarrow Y$ is, up to homotopy, the Hopf map $S^{3} \rightarrow S^{2}$ as we see from the commutative diagram

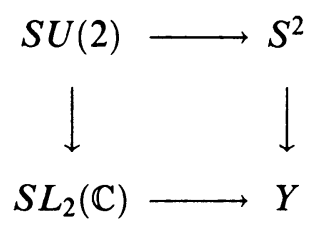

in which the vertical maps are homotopy equivalences. With the above choice of coordinates $t_{i}$, the right vertical map in this diagram is the canonical inclusion $S^{2} \subset S C^{2}$ as is easily verified. Since $\psi$ represents the generator of $\pi_{4}(H)=$ $\pi_{4}\left(S L_{2}(\mathbb{C})\right)$, the composition $S^{4} \stackrel{\psi}{\longrightarrow} S L_{2}(\mathbb{C}) \rightarrow Y$ represents the generator of $\pi_{4}\left(S C^{2}\right)=\pi_{4}\left(S^{2}\right)=\mathbb{Z} / 2 \mathbb{Z}$.

Taking coordinate rings of the spaces in (6.2) or, more precisely, in

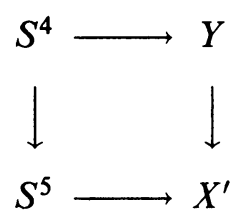

gives us, after flipping the diagram over, the diagram of rings

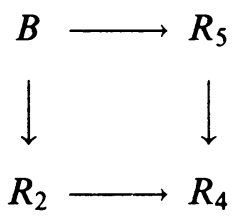

where $R_{n}=\mathbb{C}\left[x_{0}, \ldots, x_{n}\right] /\left(x_{0}^{2}+\cdots+x_{n}^{2}-1\right)$ so that $R_{2}$ is the coordinate ring of $Y$ and $R_{4}$ and $R_{5}$ are rings of functions on $S^{4}$ and $S^{5}$. 
Let $R^{\prime}$ be the pullback of

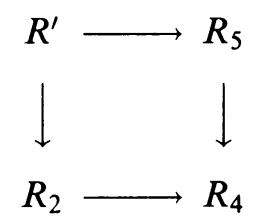

and let $R \subset R^{\prime}$ be any subring finitely generated over $\mathbb{C}$ and containing the image of $B \rightarrow R^{\prime}$.

Let $P$ be the rank 1 projective $B$-module considered in Theorem 6.3 and let $Q=R \otimes_{B} P$. Then

Theorem 6.7. $Q \oplus Q^{-1}$ is not free but $Q \oplus Q^{-1} \oplus B$ is free.

Proof. The second assertion follows immediately from Theorem 6.3. For the first assertion it will suffice to consider $Q^{\prime}=R^{\prime} \otimes_{B} P$. We can regard $R^{\prime}$ as a ring of functions on the pushout $Z$ of the diagram

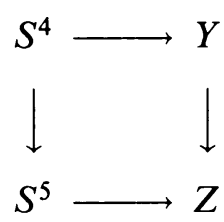

From diagram (6.3) we see that $Y \rightarrow X^{\prime}$ factors as $Y \rightarrow Z \rightarrow X^{\prime}$ and the map $B \rightarrow R^{\prime}$ is obtained from $B \rightarrow C\left(X^{\prime}\right) \rightarrow C(Z)$. The inclusions $S U(2) \subset S U(3)$ and $S L_{2}(\mathbb{C}) \subset S L_{3}(\mathbb{C})$ give us the diagram

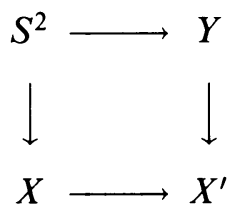

The top and bottom arrows are homotopy equivalences and we have seen in $\S 3$ that the left vertical map induces an isomorphism of $H^{2}(-, \mathbb{Z})$. The same is therefore true of the right vertical map. It is also true of $Y \rightarrow Z$ since $Z$ is obtained from $Y$ by attaching 5-cells (the two hemispheres of $S^{5}$ ). Therefore, the two maps $X \rightarrow X^{\prime} \leftarrow Z$ induce isomorphisms of $H^{2}(-, \mathbb{Z})$.

Let $L^{\prime}$ be the line bundle on $X^{\prime}$ corresponding to $C\left(X^{\prime}\right) \otimes_{B} P$. Then $L^{\prime}$ induces the bundle $L$ of Lemma 6.4 on $X$. The corresponding cohomology class $c_{1}\left(L^{\prime}\right) \in H^{2}\left(X^{\prime}, \mathbb{Z}\right)$ maps to $c_{1}(L) \in H^{2}(X, \mathbb{Z})$. Since $c_{1}(L)$ generates $H^{2}(X, \mathbb{Z})$ by Lemma 6.4 and the results of $\S 3$, we see that $c_{1}\left(L^{\prime}\right)$ generates $H^{2}\left(X^{\prime}, \mathbb{Z}\right)$.

Let $M$ be the line bundle on $Z$ corresponding to $C(Z) \otimes_{R^{\prime}} Q^{\prime}=C(Z) \otimes_{B} P$, where $Q^{\prime}=R^{\prime} \otimes_{B} P$ as above. Then $M$ is the pullback of $L^{\prime}$ under $Z \rightarrow X^{\prime}$ so $c_{1}(M)$ generates $H^{2}(Z, \mathbb{Z})$.

To show that $Q^{\prime} \oplus Q^{\prime-1}$ is nontrivial, it will suffice to show that $M \oplus M^{-1}$ is nontrivial. By Lemma 3.1, it is enough to show that $c_{1}(M)$ is not spherical. Now the maps $S^{2} \rightarrow Y \rightarrow Z$ induce isomorphisms of $H^{2}(-, \mathbb{Z})$ so $c_{1}(M)$ maps to a generator of $H^{2}\left(S^{2}, \mathbb{Z}\right)$. If $c_{1}(M)$ is spherical, then by Lemma 3.2, $\pi_{4}\left(S^{2}\right) \rightarrow \pi_{4}(Z)$ will be injective and therefore $\pi_{4}(Y) \rightarrow \pi_{4}(Z)$ will also be injective. But we have seen that the map $S^{4} \rightarrow Y$ in diagram (6.5) represents 
the generator of $\pi_{4}(Y)=\mathbb{Z} / 2 \mathbb{Z}$, and the composition $S^{4} \rightarrow Y \rightarrow Z$ is trivial since it factors through $S^{5}$ which has $\pi_{4}\left(S^{5}\right)=0$.

To complete the proof of Theorem 1(3) we must show that $R$ can be chosen finitely generated and rational. Since $R$ was any sub- $\mathbb{C}$-algebra of the pullback $R^{\prime}$ which contains the image of $B$ we can choose $R$ finitely generated.

Lemma 6.8. The map $R_{2} \rightarrow R_{4}$ in diagram (6.4) is injective.

Proof. We have $R_{2}=\mathbb{C}[p, q, r, s] /(p s-q r, q-r-1)$ and

$$
R_{4}=\mathbb{C}[b, \bar{b}, c, \bar{c}, r] /\left(r^{2}+b \bar{b}+c \bar{c}-1\right)
$$

in notations used above. The map $R_{2} \rightarrow R_{4}$ is given by $S^{4} \stackrel{\psi}{\longrightarrow} S L_{2}(\mathbb{C}) \rightarrow Y$ and sends $p, q, r, s$ to the four products of the form $x y$ where $x$ is an entry from the first column of $\psi$ and $y$ is an entry from the second column of $\psi$. Map $R_{4}$ to $\mathbb{C}[b, c]$ by sending $r$ to 1 and $\bar{b}$ and $\bar{c}$ to 0 . The composition $R_{2} \rightarrow R_{4} \rightarrow \mathbb{C}[b, c]$ sends $p, q, r, s$ to the four products formed from the matrix

$$
\left(\begin{array}{cc}
-b^{2} & -b c-1 \\
-b c+1 & -c^{2}
\end{array}\right)
$$

so the image is the subring generated by $1, b^{2} c^{2}, b^{2}(b c+1)$, and $c^{2}(b c-1)$. Let $D$ be the integral closure of the image and let $K$ be the quotient field of $D$. Then $b c \in D$ so $(b c+1)^{-1}$ and $(b c-1)^{-1}$ lie in $K$. Therefore $b^{2}$ and $c^{2}$ lie in $K$ and hence so do $b$ and $c$. This shows that $K=\mathbb{C}(b, c)$. Since $R_{2}$ is a domain of dimension 2 , the map $R_{2} \rightarrow \mathbb{C}[b, c]$ must be injective otherwise the image, and hence $K$, would have transcendence degree $\leq 1$.

Referring back to the definition (6.4) of $R^{\prime}$, we now see that $R^{\prime} \rightarrow R_{5}$ is injective. The elements $x_{0}$ and $x_{0} x_{i}$ lie in the kernel of $R_{5} \rightarrow R_{4}$ and so lie in $R^{\prime}$. If we choose $R$ to contain these elements, it is clear that $R$ will have the some quotient field as $R_{5}$ and so will be rational over $\mathbb{C}$.

\section{Nonliftable Determinants (REAl CASE)}

In this section, we will give an example to show that the map $[X, B S O(n)] \rightarrow$ $[X, B O(n)]$ is not injective in general. This problem turns out to be equivalent to a lifting problem for determinants. Another version of this result will be discussed later in $\S 9$.

Let $C(X)$ be the ring of continuous real functions on $X$. If $E$ is a vector bundle over $X$, we define an action of the group $C(X)^{*}$ on the fiber of $[X, B S O(n)] \rightarrow[X, B O(n)]$ over $E$ as follows. A preimage of $E$ consists of a pair $(E, \varphi)$ where $\varphi: \bigwedge^{n} E \approx \mathscr{O}, \mathscr{O}$ being the trivial line bundle on $X$. If $\beta \in \operatorname{Aut}(\mathscr{O})=C(X)^{*}$, define $\beta \circ(E, \varphi)=(E, \beta \varphi)$. If $(E, \psi)$ lies in the same fiber, we have $(E, \psi)=\beta \circ(E, \varphi)$ with $\beta=\psi \varphi^{-1} \in \operatorname{Aut}(\mathscr{O})$. Therefore the action of $C(X)^{*}$ is transitive on the fibers.

By taking determinants of endomorphisms of $E$ fiberwise we get a determinant map det: $\operatorname{End}(E) \rightarrow C(X)$. Two pairs $(E, \varphi)$ and $(E, \beta \varphi)$ represent the same element of $[X, B S O(n)]$ if and only if there is an automorphism $\alpha$ of $E$ such that $\varphi \circ \bigwedge^{n} \alpha=\beta \varphi$. Now $\bigwedge^{n} \alpha=\operatorname{det}(\alpha)$ in $\operatorname{Aut}\left(\bigwedge^{n} E\right)=C(X)^{*}$ so the requirement is that $\operatorname{det}(\alpha)=\beta$. Therefore the fiber over $E$ is the cokernel of $\operatorname{det}: \operatorname{Aut}(E) \rightarrow C(X)^{*}$. 
Let $C(X)_{0}^{*}$ be the subgroup of $f \in C(X)^{*}$ which are homotopic to 1 as maps into $\mathbb{R}^{*}$. If $f \in C(X)_{0}^{*}$, we can write $f=g^{n}$ for some $g \in C(X)^{*}$. This is clear in the real case since $f>0$. In the complex case we can lift $f: X \rightarrow \mathbb{C}$ to the $n$-fold covering $\mathbb{C} \rightarrow \mathbb{C}$ (where $z \rightarrow z^{n}$ ) since $f$ is homotopic to a constant. If $g \in C(X)^{*}$, the map $g: E \rightarrow E$ given by multiplication by $g$ has $\operatorname{det}(g)=g^{n}$ where $n=\operatorname{rk} E$, showing that the subgroup $C(X)_{0}^{*}$ lies in the image of $\operatorname{Aut}(E)$. This shows that the action of $C(X)^{*}$ on $[X, B S O(n)]$ factors through $C(X)^{*} / C(X)_{0}^{*}=[X, O(1)]$.

Lemma 7.1. Let $E$ be a real vector bundle on $X$ lying in the image of

$$
[X, B S O(n)] \rightarrow[X, B O(n)] .
$$

Then the fiber of the map $[X, B S O(n)] \rightarrow[X, B O(n)]$ over $E$ is isomorphic to the cokernel of $\operatorname{det}: \operatorname{Aut}(E) \rightarrow[X, O(1)]=[X,\{ \pm 1\}]$.

For a version applicable to nonorientable bundles see Corollary 9.5 below.

Corollary 7.2. If $E$ is as in Lemma 7.1, then $E$ has a unique preimage in $[X, B S O(n)]$ if and only if $\operatorname{det}: \operatorname{Aut}(E) \rightarrow[X,\{ \pm 1\}]$ is onto.

The following example of Ojanguren shows that the determinant need not be onto.

Theorem 7.3 [O, §4]. Let $E$ be the (real) tangent bundle to $S^{2 n}$. Then the preimage of $E$ in $\left[S^{2 n}, B S O(2 n)\right]$ consists of two distinct elements.

Proof. The two liftings $(E, \varphi)$ and $(E, \psi)$ to $\left[S^{2 n}, B S O(2 n)\right]$ consist of $E$ with its two possible orientations. If $\chi(E, \varphi) \in H^{2 n}\left(S^{2 n}\right)$ denotes the Euler class, it is well known [MS] that $\chi(E, \psi)=-\chi(E, \varphi)$ so $(E, \psi) \neq(E, \varphi)$ since $\chi(E, \varphi) \neq 0$.

Corollary 7.4. If $E$ is the real tangent bundle to $S^{2 n}$, there is no automorphism $\alpha$ of $E$ with $\operatorname{det}(\alpha)=-1$.

Corollary 7.5. Let $A=\mathbb{R}\left[x_{0}, \ldots, x_{2 n}\right] /\left(x_{0}^{2}+\cdots+x_{2 n}^{2}-1\right)$ and let $P$ be the projective A-module defined by the unimodular row $\left(x_{0}, \ldots, x_{2 n}\right)$. Then if $f$ is any automorphism of $P, \operatorname{det}(f)>0$.

This follows from the well-known fact that $P$ induces the bundle $E$ [SV] and $A^{*}=\mathbb{R}^{*}$ [SM, Lemma 9.1].

Remark. There is no corresponding result for endomoprhisms of $P$. For example, let $A=\mathbb{R}[x, y, z] /\left(x^{2}+y^{2}+z^{2}-1\right)$, so that $P$ has generators $\alpha, \beta$, $\gamma$ with the relation $x \alpha+y \beta+z \gamma=0$. Let $f: P \rightarrow P$ by

$$
f(\alpha)=\left(1-z^{2}\right) \alpha+2 x z \gamma, \quad f(\beta)=\left(1-z^{2}\right) \beta+2 y z \gamma, \quad f(\gamma)=\left(z^{2}-1\right) \gamma .
$$

Then it is easy to see that $\operatorname{det}(f)=-\left(1-z^{2}\right)^{2} \leq 0$. Here $f$ fails to be an isomorphism on the fibers over $z= \pm 1$.

\section{NONLIFTABLE DETERMINANTS (COMPLEX CASE)}

The first two results of $\S 7$ have immediate analogues in the complex case with exactly the same proofs. 
Lemma 8.1. Let $E$ be a complex vector bundle on $X$ lying in the image of $[X, B S U(n)] \rightarrow[X, B U(n)]$. Then the fiber of the map $[X, B S U(n)] \rightarrow$ $[X, B U(n)]$ over $E$ is isomorphic to the cokernel of $\operatorname{det}: \operatorname{Aut}(E) \rightarrow[X, U(1)]$ $=\left[X, S^{1}\right]$.

Corollary 8.2. If $E$ is as in the Lemma 7.1, then $E$ has a unique preimage in $[X, B S U(n)]$ if and only if $\operatorname{det}: \operatorname{Aut}(E) \rightarrow\left[X, S^{1}\right]$ is onto.

It does not seem to be quite as easy to prove results like Theorem 7.3 in the complex case since there is no obvious analogue of the Euler class which we can use. However, similar results also hold in this case.

Recall that $\left[S^{n}, B U(n)\right]=\pi_{n}(B U(n))=\pi_{n-1}(U(n))$. The element of $\pi_{n-1}(U(n))$ corresponding to a bundle is given by a clutching function on the equator $S^{n-1}$ of $S^{n}$ [HFB, SFB].

Theorem 8.3. Let $E \rightarrow S^{n}$ be either the rank 2 complex on $S^{4}$ corresponding to the generator of $\pi_{4} B U(2)=\mathbb{Z}$, or the rank 2 complex bundle on $S^{5}$ corresponding to the generator of $\pi_{5} B U(2)=\mathbb{Z} / 2 \mathbb{Z}$. Let $X=S^{n} \times S^{1}$ and let $p^{*} E$ be the pullback of $E$ to $X$ by the projection $p: X \rightarrow S^{n}$. Let $q: X \rightarrow S^{1}$ be the other projection. Then there is no automorphism $\alpha$ of $p^{*} E$ with $\operatorname{det}(\alpha)=q$.

Algebraic descriptions of these bundles will be given below.

Proof. Let $E$ be the given bundle with clutching function $c$. We can assume that $c: S^{n-1} \rightarrow S U(2)$ since $\pi_{n-1}(S U(2)) \rightarrow \pi_{n-1}(U(2))$ is an isomorphism for $n \geq 3$. We can also assume that $c(*)=1$ where $*$ is a basepoint of $S^{n-1}$. Let $U$ be the upper hemisphere of $S^{n}$ and let $V$ be the lower hemisphere. Write the points of $U \times \mathbb{C}^{2}$ as $(u, x)_{U}$ and those of $V \times \mathbb{C}^{2}$ as $(v, x)_{V}$. Then $E=(U \times$ $\left.\mathbb{C}^{2}\right) \cup\left(V \times \mathbb{C}^{2}\right)$ with the identification $(u, x)_{U}=(u, c(u) x)_{V}$ for $u \in S^{n-1}$ and therefore $p^{*} E=\left(U \times S^{1} \times \mathbb{C}^{2}\right) \cup\left(V \times S^{1} \times \mathbb{C}^{2}\right)$ with $(u, s, x)_{U}=(u, s, c(u) x)_{V}$ for $u \in S^{n-1}$. Now if $\alpha$ is an automorphism of $p^{*} E$, its restriction to $U \times$ $S^{1} \times \mathbb{C}^{2}$ takes the form $\alpha(u, s, x)_{U}=(u, s, \beta(u, s) x)_{U}$ and its restriction to $V \times S^{1} \times \mathbb{C}^{2}$ takes the form $\alpha(u, s, x)_{V}=(u, s, \gamma(u, s) x)_{V}$, where $\beta(u, s)$ and $\gamma(u, s)$ lie in $G L_{2}(\mathbb{C})$. For $u \in S^{n-1}$, compatibility with the identifications requires that $\alpha(u, s, x)_{U}=\alpha(u, s, c(u) x)_{V}$. Therefore $(u, s, \beta(u, s) x)_{U}=$ $(u, s, \gamma(u, s) c(u) x)_{V}$. But $(u, s, \beta(u, s) x)_{U}=(u, s, c(u) \beta(u, s) x)_{V}$ which gives us the relation

$$
\gamma(u, s) c(u)=c(u) \beta(u, s) \quad \text { for } u \in S^{n-1} .
$$

Suppose now that $\operatorname{det}(\alpha)=q$. Then $\operatorname{det} \beta(u, s)=s=\operatorname{det} \gamma(u, s)$. Let $\tilde{s}$ be the diagonal matrix $\left(\begin{array}{ll}s & 0 \\ 0 & 1\end{array}\right)$ in $U(2)$. Then we have $\beta(u, s)=\tilde{s} \varphi(u, s)$ and $\gamma(u, s)=\tilde{s} \psi(u, s)$ where $\varphi(u, s)$ and $\psi(u, s)$ lie in $S L_{2}(\mathbb{C})$. The relation (8.1) becomes

$$
\tilde{s} \psi(u, s) c(u)=c(u) \tilde{s} \varphi(u, s) \quad \text { for } u \in S^{n-1} .
$$

Now $\varphi(u, s)$ is defined on $U \times S^{1}$ which has the homotopy type of $S^{1}$. Since $S L_{2}(\mathbb{C})$ is simply connected, $\varphi(u, s) \simeq 1$ (the constant map) and similarly, $\psi(u, s) \simeq 1$. Therefore, (8.2) shows that the two maps $S^{n-1} \times S^{1} \rightarrow G L_{2}(\mathbb{C})$ sending $(u, s)$ to $\tilde{s} c(u)$ and and $c(u) \tilde{s}$ are homotopic. This is equivalent to the assertion that $\theta: S^{n-1} \times S^{1} \rightarrow G L_{2}(\mathbb{C})$ by $\theta(u, s)=[\tilde{s}, c(u)]$ is homotopic to 1 . Conversely, if this is so, $\alpha$ exists. It suffices to set $\varphi(u, s)=1$, define $\psi(u, s)$ 
on $S^{n-1} \times S^{1}$ by $(8.2)$, and observe that $\psi(u, s) \simeq 1$ so $\psi(u, s)$ extends to $V \times S^{1}$.

To prove the theorem, it will therefore suffice to show that $\theta$ is not homotopic to the constant map in the two cases considered in the theorem. Note that $\theta$ maps $S^{n-1} \times S^{1}$ to $S U(2) \subset S L_{2}(\mathbb{C})$. Since $S U(2) \hookrightarrow S L_{2}(\mathbb{C})$ is a homotopy equivalence and $G L_{2}(\mathbb{C})$ is homeomorphic to $S L_{2}(\mathbb{C}) \times \mathbb{C}^{*}$, it will be enough to show that $\theta$ is not homotopically trivial as a map into $S U(2)$.

Now $\theta$ sends $S^{n-1} \vee S^{1}=\left(S^{n-1} \times 1\right) \cup\left(* \times S^{1}\right)$ to 1 . Since $S U(2)=S^{3}$ is 2-connected, it will suffice, by Lemma 9.1 and Corollary 9.2, to show that $\theta$ is nontrivial in $\left[\left(S^{n-1} \times S^{1}\right) /\left(* \times S^{1}\right), S U(2)\right]$. But $\left(S^{n-1} \times S^{1}\right) /\left(* \times S^{1}\right) \simeq S^{n-1} \vee S^{n}$ (see the remark below) and $\left[S^{n-1} \vee S^{n}, S U(2)\right]=\left[S^{n-1}, S U(2)\right] \times\left[S^{n}, S U(2)\right]$. The image of $\theta$ in $\left[S^{n-1}, S U(2)\right]$ is trivial as observed above, so it will suffice to show that the map $\theta: S^{n}=\left(S^{n-1} \times S^{1}\right) /\left(S^{n-1} \vee S^{1}\right) \rightarrow S U(2)$ is nontrivial.

Remark. The fact that $\left(S^{p} \times S^{q}\right) /\left(* \times S^{q}\right) \simeq S^{p} \vee S^{p+q}$ can be seen as follows. By [Br, 4.7], $S^{p} \times S^{q}=e^{0} \cup e^{p} \cup e^{q} \cup e^{p+q}$ so $\left(S^{p} \times S^{q}\right) /\left(* \times S^{q}\right)=e^{0} \cup e^{p} \cup e^{p+q}$ with $e^{0} \cup e^{p}=S^{p} \times *$. Since $S^{p}=S^{p} \times * \hookrightarrow\left(S^{p} \times S^{q}\right) /\left(* \times S^{q}\right) \rightarrow S^{p}$ (by the projection) is the identity map, the attaching map $e^{p+q-1} \rightarrow S^{p}$ for $e^{p+q}$ must be homotopic to the constant map. Now apply [Br, 7.5.5].

Recall that the group $S U(2)$ is isomorphic to the group $S^{3}$ of unit quaternions, the matrix $\left(\begin{array}{cc}a & b \\ -b & \frac{a}{a}\end{array}\right)$ corresponding to the quaternion $a+b j$. It is easy to check that conjugation by $\tilde{s}$ on $S U(2)$ corresponds to the action on $S^{3}$ sending $a+b j$ to $a+s b j$. Therefore, if we let $c(u)$ in $S U(2)$ correspond to $a+b j=a(u)+b(u) j$ in $S^{3}$, the map $\theta: S^{n-1} \times S^{1} \rightarrow S U(2)=S^{3}$ is given by $\theta(u, s)=(a+s b j)(a+b j)^{-1}=1+(s-1) b j(a+b j)^{-1}=1+(s-1) b j(\bar{a}-b j)=$ $1+(s-1)|b|^{2}+(s-1) a b j$. In particular, the $i$-coordinate of $\theta(u, s)$ is $|b|^{2} \operatorname{Im} s$.

Choose the equator of $S^{n}=\left(S^{n-1} \times S^{1}\right) /\left(S^{n-1} \vee S^{1}\right)$ to be given by the image of $S^{n-1} \times-1$. This clearly contains the image $*$ of $S^{n-1} \vee S^{1}=\left(S^{n-1} \times 1\right) \cup$ $\left(* \times S^{1}\right)$. We choose the upper hemisphere to be given by $\operatorname{Im} s \geq 0$ and the lower hemisphere to be given by $\operatorname{Im} s \leq 0$. If we choose the upper and lower hemispheres of $S^{3}$ to be given by the $i$-coordinate, it follows that $\theta^{\prime}$ maps the upper hemisphere of $S^{n}$ to the upper hemisphere of $S^{3}$ and similarly for the lower hemispheres. Therefore $\theta^{\prime}$ is the suspension of the map $\eta: S^{n-1} \rightarrow S^{2}$ given by restricting $\theta^{\prime}$ to the equators, i.e., by setting $s=-1$. This map sends $u$ to $1-2|b|^{2}-2 a b j=|a|^{2}-|b|^{2}-2 a b j$, lying in the unit sphere of $\mathbb{R}+\mathbb{R} j+\mathbb{R} k$. This is exactly the formula for the Hopf map $h: S^{3} \rightarrow S^{2}$ so it follows that $\theta^{\prime}$ is the suspension of the composition $S^{n-1} \stackrel{c}{\longrightarrow} S^{3} \stackrel{h}{\longrightarrow} S^{2}$ or, in other words, $\theta^{\prime}=\Sigma h \circ \Sigma c$.

Recall that if we define $\pi_{n}=\operatorname{colim} \pi_{n+k}\left(S^{k}\right)$ as $k \rightarrow \infty$, then $\bigoplus \pi_{n}$ is an algebra under composition, $\pi_{1}=\mathbb{Z} / 2 \mathbb{Z}$, generated by $\eta$ the image of the Hopf map $h$, and $\pi_{2}=\mathbb{Z} / 2 \mathbb{Z}$, generated by $\eta^{2}=\eta \circ \eta$ [Hu, Chapter XI, $\S 15$ ]. For the first bundle considered in Theorem 8.3 , we have $c=1$ so the image of $\theta^{\prime}$ in $\pi_{1}$ is the generator and hence is nontrivial. For the second bundle considered, $c=\Sigma h$ [Hu, loc. cit.] so the image of $\theta^{\prime}$ in $\pi_{2}$ is the generator $\eta^{2}$ which is also nontrivial.

Corollary 8.4. If $E$ is as in Theorem 8.3, the preimage of $E$ in $\left[S^{n} \times S^{1}, B S U(2)\right]$ has 2 elements. 
Proof. As observed above, $E$ has an $S U(2)$ structure so the preimage is nonempty. We have $\left[S^{n} \times S^{1}, S^{1}\right]=\mathbb{Z}$ generated by $q$. Since $q^{2}$ is the determinant of multiplication by $q$ on $E$, the result follows from Lemma 8.1 and Theorem 8.3 .

We can give algebraic descriptions of the two bundles as follows.

(1) Let $A=\mathbb{R}\left[t, x_{0}, \ldots, x_{3}\right] /\left(t^{2}+x_{0}^{2}+\cdots+x_{3}^{2}-1\right)$ be the coordinate ring of $S^{4}$ considered as the unit sphere of $\mathbb{R} \times \mathbb{H}$. Define an idempotent endomorphism $\rho$ of the free module $\left(\mathbb{H} \otimes_{\mathbb{R}} A\right)^{2}$ by $\rho\left(y_{1}, y_{2}\right)=\left(y_{1}, y_{2}\right) M$ where $M$ is the matrix

$$
\frac{1}{2}\left(\begin{array}{cc}
1-t & q \\
\bar{q} & 1+t
\end{array}\right)
$$

with $q=x_{0}+x_{1} i+x_{2} j+x_{3} k$. The image of $\rho$ is a rank 1 projective module $P$ over $\mathbb{H} \otimes_{\mathbb{R}} A$. We regard $P$ as a rank 2 projective module over the subring $R=\mathbb{C} \otimes_{\mathbb{R}} A=\mathbb{C}\left[t, x_{0}, \ldots, x_{3}\right] /\left(t^{2}+x_{0}^{2}+\cdots+x_{3}^{2}-1\right)$. By [SV, p. 276] and [SFB, 20.4], this has the Hopf bundle $S^{7} \rightarrow S^{4}$ as associated principal bundle. This corresponds to the generator of $\pi_{4} B U(2)=\mathbb{Z}$.

(2) Let $R=\mathbb{C}\left[x_{0}, \ldots, x_{5}\right] /\left(x_{0}^{2}+\cdots+x_{5}^{2}-1\right)$ be the complex coordinate ring of $S^{5}$. Set $z_{1}=x_{0}+i x_{1}, z_{2}=x_{2}+i x_{3}, z_{3}=x_{4}+i x_{5}$ and let $P$ be the projective $R$-module defined by the unimodular row $\left(z_{1}, z_{2}, z_{3}\right)$. By [ST, $\mathrm{p}$. 433], $P$ defines a nontrivial vector bundle on $S^{5}$ as required.

Corollary 8.5. Let $R$ and $P$ be as in one of the two examples just cited. Then there is no automorphism $\alpha$ of $P\left[t, t^{-1}\right]=R\left[t, t^{-1}\right] \otimes_{R} P$ such that $\operatorname{det}(\alpha)=1$.

\section{BASED HOMOTOPIES}

I will give here a brief discussion of the relation between free homotopies and basepoint preserving homotopies with emphasis on the case of classifying spaces. This problem is closely related to the one discussed in $\S 7$.

Let $X$ be a $C W$-complex and $Y$ a subcomplex of $X$. As usual we define $X / Y$ by taking the disjoint union $X \cup *$ of $X$ with a point and identifying all points of $Y$ with $*$. Therefore $X / Y$ is $X \cup *$ if $Y$ is empty and otherwise is the space obtained by collapsing $Y$ to a point.

Definition. If $X$ and $Y$ are spaces with basepoint let $\left[X, Y_{0}\right]$ denote the set of homotopy classes of maps of $X$ to $Y$ which preserve the basepoint, the homotopies also being required to preserve the basepoint (notation as in [HFB, Chapter $1, \S 4])$. Let $[X, Y]$ denote, as above, the set of homotopy classes of maps of $X$ to $Y$ which are not required to preserve the basepoint.

Up to now we have been considering $[X, Y]$. The object of this section is to study the relation between $[X, Y]$ and $[X, Y]_{0}$. There is clearly a map $[X, Y]_{0} \rightarrow[X, Y]$. In general this is neither injective nor surjective.

Lemma 9.1. Let $Z$ be an $n$-connected space. Then

(1) If $\operatorname{dim} Y \leq n$, then $[X / Y, Z]_{0} \rightarrow[X, Z]$ is surjective.

(2) If $\operatorname{dim} Y \leq n-1$, then $[X / Y, Z]_{0} \rightarrow[X, Z]$ is bijective.

Proof. If $f: X \rightarrow Z$ and $\operatorname{dim} Y \leq n$, we can deform $f \mid Y$ to the basepoint. By the homotopy extension theorem, the homotopy can be extended to one of $f$ showing that the map is onto. Suppose that $\operatorname{dim} Y \leq n-1$. Let $f, g: X / Y \rightarrow$ $Z$ preserving the basepoint, and suppose that $f \simeq g$ as maps $X \rightarrow Z$ via 
a homotopy $h: X \times I \rightarrow Z$. This factors through $h: W \rightarrow Z$ where $W=$ $(X \times I) /(Y \times 0) \cup(Y \times 1)$. Let $V=(Y \times I) /(Y \times 0) \cup(Y \times 1)$ be the image of $Y \times I$ in $W$. Since this has dimension $\leq n$, there is a homotopy of $h^{\prime} \mid V$ to the constant map keeping the basepoint fixed. Patching this with the constant homotopy on $X \times 0$ and $X \times 1$ gives a homotopy of $(X \times 0) \cup(X \times 1) \cup V$. Extend this to a homotopy $H: W \times I \rightarrow Z$ of $h^{\prime}$. Then $H \mid W \times 1$ gives the required homotopy of $f$ and $g$ as maps of $X / Y$ to $Z$.

Corollary 9.2 [BR, p. 230, Exercise 10]. If $Z$ is 0 -connected, then $[X, Z]_{0} \rightarrow$ $[X, Z]$ is surjective. If $Z$ is 1-connected, it is bijective.

We can get more precise information in the non-simply-connected case by defining a right action of $\pi_{1}(Z)$ on $[X, Z]_{0}$. Let $x_{0}$ and $z_{0}$ be the basepoints of $X$ and $Z$. Let $f: X \rightarrow Z$ with $f\left(x_{0}\right)=x_{0}$ and let $\omega$ be a loop at $z_{0}$. Then $t \mapsto \omega(t)$ is a homotopy of $f \mid x_{0}$ which we extend to a homotopy $h: X \times I \rightarrow Z$ with $h \mid X \times 0=f$ and $f \mid x_{0} \times I=\omega$. Let $g=f \mid X \times 1$. We write $f \circ \omega=g$ for convenience although this depends on the choice of $h$.

It is not hard to see that we can define a right action of $\pi_{1}(Z)$ on $[X, Z]_{0}$ by setting $[f] \circ[\omega]=[g](\mathrm{cf} .[\mathrm{SFB}, 16.1])$, and that $[X, Z]_{0} / \pi_{1}(Z) \rightarrow[X, Z]_{0}$ is bijective if $Z$ is 0 -connected. For the present purposes we will only need the definition of $f \circ \omega$.

By Corollary 9.2, $[X, B S O(n)],[X, B U(n)]$, and $[X, B S U(n)]$ will be the same whether we consider $[,]_{0}$ or $[$,$] . The following result explains the$ remaining situation.

Theorem 9.3. $[X, B O(n)]_{0}$ classifies isomorphism classes of real rank $n$ vector bundles on $X$ with a specified orientation on the fiber over the basepoint.

Corollary 9.4. If $X$ is 1-connected, then $[X, B S O(n)] \rightarrow[X, B O(n)]_{0}$ is bijective.

This follows from the fact that all vector bundles on a simply connected space are orientable so orienting the fiber over the basepoint is equivalent to orienting the bundle. It is also clear directly since each map $X \rightarrow B O(n)$ has a unique lift to $B S O(n)$ preserving the basepoint.

The following gives a generalization of Lemma 7.1 to the case of not necessarily orientable bundles.

Corollary 9.5. Let $X$ be a connected space and let $E$ be a real rank $n$ vector bundle on $X$, classified by a map $f: X \rightarrow B O(n)$. Then $E$ has an automorphism with determinant -1 if and only if the preimage of $[f]$ under $[X, B O(n)]_{0} \rightarrow[X, B O(n)]$ consists of a single element.

Proof. Choose an orientation for the fiber of $E$ over the basepoint. An automorphism with determinant -1 will reverse this orientation showing that the two possible orientations give isomorphic bundles. Conversely, an automorphism of the bundle which reverses the orientation over the basepoint will necessarily have a negative determinant $d$ at all points since $X$ is connected. Dividing the automorphism by $|d|^{1 / n}$ gives the required automorphism.

Proof of Theorem 9.3. Let $f_{0}, f_{1}: X \rightarrow B O(n)$ and let $E_{i}=f_{1}^{*} \mathscr{E}$ where $\mathscr{E}$ is the canonical bundle on $B O(n)$. If $f_{0} \simeq f_{1}$, we get an isomorphism $E_{0} \approx E_{1}$ as follows. Let $h: X \times I \rightarrow B O(n)$ be a homotopy between $f_{0}$ and $f_{1}$. By a 
standard fact about bundles [HFB, SFB], there is an isomorphism $\psi: E \times I \approx$ $h^{*} \mathscr{E}$ for some bundle $E$ on $X$. Let $\psi_{i}$ be the restriction of $\psi$ to $E \times i$ for $i=0,1$. Then $\psi_{i}: E \approx E_{i}$ so $\psi_{1} \psi_{0}^{-1}: E_{0} \approx E_{1}$. Write $F_{i}$ for the fiber of $E_{i}$ over the basepoint $x_{0}$ and $F$ for the fiber of $E$. Let $h^{\prime}$ be the restriction of $h$ to $x_{0} \times I$. Let $\varphi$ be the restriction of $\psi: E \times I \approx h^{*} \mathscr{E}$ to $\varphi: F \times I \approx h^{\prime * \mathscr{E}}$. Then $\psi_{1} \psi_{0}^{-1}$ restricts to the isomorphism $\varphi_{1} \varphi_{0}^{-1}: F_{0} \approx F_{1}$, where $\varphi_{i}$ is the restriction of $\varphi$ to $F \times i$ for $i=0,1$. A different choice $\psi^{\prime}$ for $\psi$ leads to a new map $\varphi^{\prime}: F \times I \approx h^{\prime *} \mathscr{E}$ and we can write $\varphi^{\prime}=\varphi \sigma$ where $\sigma$ is an automorphism of $F \times I$ over $I$. It follows that $\varphi_{1}^{\prime} \varphi_{0}^{\prime-1}=\varphi_{1} \sigma_{1} \sigma_{0}^{-1} \varphi_{0}^{-1}$. Now $F=\mathbb{R}^{n}$ so $\sigma$ has the form $\sigma(x, t)=(\gamma(t) x, t)$ where $\gamma: I \rightarrow G L_{n}(\mathbb{R})$. Therefore $\sigma_{1} \sigma_{0}^{-1}=\gamma(1) \gamma(0)^{-1} \simeq \gamma(0) \gamma(0)^{-1}=1$ so $\sigma_{1} \sigma_{0}^{-1}$ preserves orientation. This shows that the sign $\varepsilon(h)$ of the determinant of $\varphi_{1} \varphi_{0}^{-1}: F_{0} \approx F_{1}$ depends only on $h$ and not on the choice of $\psi$. To find the value of $\varepsilon(h)$ it is sufficient to consider the case where $X$ is a point.

Lemma 9.6. Let $h: I \rightarrow B O(n)$ send 0 and 1 to the basepoint $*$. Then $\varepsilon(h)=1$ if and only if $[h]=1$ in $\pi_{1}(B O(n))$.

Proof. Consider the diagram

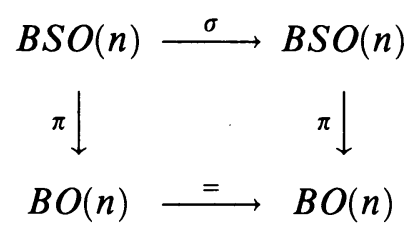

where $\sigma$ is the covering transformation. We have $\pi^{*} \mathscr{E}=\mathscr{E}^{\prime}$, the canonical bundle on $B S O(n)$ and the diagram shows that $\sigma^{*} \mathscr{E}^{\prime}=\mathscr{E}^{\prime}$. However, the orientation must be reversed otherwise it would descend to an orientation of $\mathscr{E}$ which would imply that all $O(n)$ bundles are orientable.

Let $\left\{*_{1}, *_{2}\right\}$ be the fiber of $B S O(n) \rightarrow B O(n)$ over the basepoint $*$. Let $\mathscr{F}$ be the fiber of $\mathscr{E}$ over $*$, and let $\mathscr{F}_{i}$ be the fiber of $\mathscr{E}^{\prime}$ over $*_{i}$. Then $\pi$ induces isomorphisms $\mathscr{F}_{1} \approx \mathscr{F} \approx \mathscr{F}_{2}$. The composition is given by $\sigma$ so if we orient $\mathscr{F}$ to make $\mathscr{F}_{1} \approx \mathscr{F}$ preserve orientation, it follows that $\mathscr{F} \approx \mathscr{F}_{2}$ reverses orientation.

Lift $h$ to $h^{\prime}: I \rightarrow B S O(n)$ with $h^{\prime}(0)=*_{1}$. Let $h_{i}^{\prime}=h^{\prime} \mid\{i\}$ for $i=0,1$ and similarly for $h$. Choose an isomorphism $h^{*} \mathscr{E}=h^{\prime} * \mathscr{E}^{\prime} \approx F \times I$ and orient $F$ so that $h^{\prime * \mathscr{E}}=F \times I$ preserves orientation. The diagram

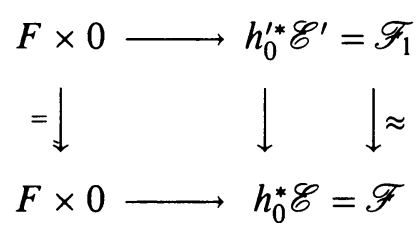

shows that $F \rightarrow h_{0}^{*} \mathscr{E}$ preserves orientation. Let $H^{\prime}(1)=*_{j}$ and consider the diagram

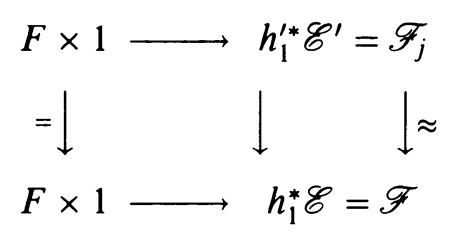


This shows that $j=1$ if and only if $F \rightarrow h_{1}^{*} \mathscr{E}$ preserves orientation. Therefore $\varepsilon(h)=1$ if and only if $h^{\prime}(1)=*_{1}$ which is equivalent to $[h]=1$ in $\pi_{1}(B O(n))$ since $\pi_{1}(B S O(n))=0$.

Now, returning to the proof of the theorem, let $\omega$ represent a nontrivial loop at $*$ in $B O(n)$. If $E$ is a bundle on $X$, we can find $f: X \rightarrow B O(n)$ with $f^{*} \mathscr{E} \approx E$. If the orientations do not agree over $*$, replace $f$ by $f \circ \omega$. This shows that $[X, B O(n)]_{0}$ maps onto the specified set of isomorphism classes, and that under this map, the action of $\pi_{1}(B O(n))$ on $[X, B O(n)]_{0}$ corresponds to reversing the orientation on the fiber over the basepoint.

To show that two maps giving the same isomorphism class are homotopic, we proceed as in [SFB, 19.3]. Let $f_{0}, f_{1}: X \rightarrow B O(n)$, write $E_{i}=f_{i}^{*} \mathscr{E}$, and let $\varphi: E_{0} \approx E_{1}$. Let $E=E_{0} \times I$ as a bundle over $X \times I$, let $Y=(X \times 0) \cup(X \times 1)$, and let $f: Y \rightarrow B O(n)$ by $f_{0}$ on $X \times 0$ and $f_{1}$ on $X \times 1$. Define $\eta: E \mid Y \approx f^{*} \mathscr{E}$ by the identity over $X \times 0$ and $\varphi$ over $X \times 1$. By [SFB, 19.2], $(f, \eta)$ extends to $(h, \psi)$ where $h: X \times I \rightarrow B O(n)$ (showing that $h: f_{0} \simeq f_{1}$ ) and $\psi: E \approx h^{*} \mathscr{E}$. Note that the isomorphism $\varphi$ is obtained from $(h, \psi)$ by the construction considered above preceding Lemma 9.6.

Suppose now that $\varphi$ preserves the orientation over the basepoint $x_{0}$. Let $\omega=h \mid x_{0} \times I$. By Lemma 9.6, $[\omega]=1$ in $\pi_{1}(B O(n))$. The homotopy $\omega \simeq 1$ can be extended, as in the proof of 9.1 , to a homotopy $h \simeq k$ fixing $f_{0}$ and $f_{1}$. This $k$ now gives the required homotopy of $f_{0}$ and $f_{1}$ fixing the basepoint.

\section{REFERENCES}

[AT] E. Artin and J. T. Tate, A note on finite ring extensions, J. Math. Soc. Japan 3 (1951), 74-77.

[B] H. Bass, Algebraic K-theory, Benjamin, New York, 1968.

[Br] R. Brown, Elements of modern topology, McGraw-Hill, Maidenhead, 1968.

[H] M. Hajja, A note on monomial automorphisms, J. Algebra 85 (1983), 243-250.

[Hu] S. T. Hu, Homotopy theory, Academic Press, New York, 1959.

[HFB] D. Husemoller, Fibre bundles, Springer-Verlag, New York 1975.

[MS] J. W. Milnor and J. D. Stasheff, Characteristic classes, Ann. of Math. Stud., no. 76, Princeton Univ. Press, Princeton, N.J., 1974.

[O] M. Ojanguren, Unités représentées par des formes quadratiques ou par des normes réduites, Lecture Notes in Math., vol. 967, Springer-Verlag, Berlin and New York, 1982, pp. 291-299.

[R] M. Raynaud, Anneaux locaux Henséliens, Lecture Notes in Math., vol. 169, Springer-Verlag, Berlin and New York, 1970.

[SCL] J-P. Serre, Corps locaux, Actualités Sci. Indust., no. 1296, Hermann, Paris, 1966.

[SFB] N. E. Steenrod, The topology of fibre bundles, Princeton Univ. Press, Princeton, N.J., 1952.

[SGA] A. Grothendieck, Revêtements étales et groupe fondamental, SGA 1, Lecture Notes in Math., vol. 224, Springer-Verlag, Berlin and New York, 1971.

[SM] R. G. Swan, Vector bundles, projective modules, and the K-theory of spheres, Proc. of the John Moore Conference, Algebraic Topology and Algebraic $K$-Theory (W. Browder, ed.), Ann. of Math. Stud., no. 113, Princeton Univ. Press, Princeton, N.J., 1987, pp. 432-522.

[SN] _ Noether's problem in Galois theory, Emmy Noether in Bryn Mawr (B. Srinivasan and J. Sally, eds.), Springer-Verlag, New York, 1983, pp. 21-40.

[ST] R. G. Swan and J. Towber, A class of projective modules which are nearly free, J. Algebra 36 (1975), 427-434. 
[SV] R. G. Swan, Vector bundles and projective modules, Trans. Amer. Math. Soc. 105 (1962), 264-277.

[SX] _ Topological examples of projective modules, Trans. Amer. Math. Soc. 230 (1977), 201-234.

Department of Mathematics, University of Chicago, Chicago, Illinois 60637

E-mail address: swan@zaphod.uchicago.edu 